\title{
Prevalence and correlates of healthy lifestyle behaviors among early cancer survivors
}

Iris M. Kanera ${ }^{1 *}$, Catherine A. W. Bolman', Ilse Mesters², Roy A. Willems', Audrey A. J. M. Beaulen and Lilian Lechner'

\begin{abstract}
Background: Healthy lifestyle behaviors have been demonstrated to be beneficial for positive health outcomes and the quality of life in cancer survivors. However, adherence to recommendations is low. More insight is needed in factors that may explain engagement in lifestyle behaviors to develop effective cancer aftercare interventions. This study assessed different factors, namely socio-demographic, cancer-related, psychological, social cognitive factors (attitude, social support, self-efficacy) and intention, in relationship to five lifestyle behaviors (smoking, physical activity, alcohol, and fruit and vegetable consumption).
\end{abstract}

Methods: Early survivors of various types of cancer were recruited from eighteen Dutch Hospitals $(n=255)$. Distal factors (socio-demographic, cancer related, psychological), proximal factors (social cognitive), intention and five lifestyle behaviors (smoking, physical activity, alcohol, fruit and vegetable consumption) were assessed through a self-reported questionnaire. Cross-sectional analyses (correlations and regression analyses) were conducted.

Results: The lifestyle of a small group (11\%) of the cancer survivors was coherent with all five health recommendations, the majority ( $>80 \%)$ adhered to two, three of four recommendations, and only few $(<7 \%)$ adhered to one or none recommendation. The highest prevalence in followed recommendations have been detected in physical activity (87.4\%), refrain from smoking (82\%), and alcohol consumption (75.4\%). There was low adherence to the fruit recommendation (54.8\%) and to the vegetable recommendation (27.4\%). Only weak associations were found between the different behaviors. Each separate lifestyle behavior was influenced by different patterns of correlates. Self-efficacy, attitude, and intention were the strongest correlates in all examined behaviors, although with various contributions, while socio-demographic, cancer-related and psychological factors provided a much smaller contribution.

Conclusions: Outcomes of engagement in healthy lifestyle behaviors were more positive in this study compared to other research in cancer survivors; however, there is room for improvements in adherence to all five lifestyle behaviors. Especially fruit consumption was poor and vegetable consumption even worse. Our findings emphasized that all examined lifestyle behaviors need to be encouraged, with taken into account that each lifestyle behavior may be influenced by a specific set of mainly social cognitive factors or intention.

Keywords: Cancer survivors, Health behaviors, Physical activity, Nutrition guidelines, Smoking, Alcohol drinking, Selfefficacy, Behavior mechanisms

\footnotetext{
* Correspondence: iris.kanera@ou.nl

${ }^{1}$ Faculty of Psychology and Educational Sciences, Open University of the

Netherlands, P. O. Box 29606401DL, Heerlen, The Netherlands

Full list of author information is available at the end of the article
} 


\section{Background}

A healthy lifestyle is of major importance for cancer survivors, since it has been shown that adherence to an increasing number of health recommendations may lower the risk of lifestyle related chronic diseases and may lead to a higher health related quality of life [1-5]. Moreover, unhealthy behaviors may have a negative impact on quality of life and cause new health problems such as cancer recurrence, new primary tumors and other chronic diseases [2, 6-11]. Health recommendations for cancer survivors include the following: achieve and maintain a healthy body weight (body mass index (BMI) within the range of 18.5 to $25.0 \mathrm{~kg} / \mathrm{m} 2$ ), engage in at least $30 \mathrm{~min}$ of moderately intense physical activity per day at five or more days weekly, eat five servings of fruit and vegetables daily, avoid or limit alcohol consumption to up to two servings per day for men and one serving per day for women, and refrain from smoking [12-14]. Previous research suggested that adherence to physical activity recommendations might be the most important lifestyle behavior associated with lower mortality and higher quality of life in cancer survivors $[10,11,15]$.

Recent research showed that cancer survivors do not adhere consistently to these health recommendations. More than half is overweight, less than half adhere to physical activity recommendations, about only one fifths adhere to fruit en vegetable recommendations, about $90 \%$ do not smoke, and approximately $90 \%$ of cancer survivors adhere to the alcohol recommendations [1, 10, $16,17]$. Broadly, similar results were found in people without a history of cancer [18-21]. Until now, research about the adherence to a combination of health behaviors showed mixed results: European studies report about $10-28 \%$ of the cancer survivors followed zero or one recommendation, about one third adhered to two, and also about one third adhered to three, and about 10$23 \%$ adhered to four recommendations [3, 4]. American studies reported even lower adherence scores to multiple health behaviors $[1,21,22]$. In comparison, research conducted in the general population among older adults indicated that most of them followed three or more lifestyle recommendations (86\%) [23], suggesting less adherence among cancer survivors compared to the general population. Considering that cancer survivors are at increased risk of cancer recurrence and lifestylerelated chronic diseases, adhering to multiple lifestyle recommendations is however very important for the health related quality of life of this specific group. This underlines the need to understand which factors explain the different health behaviors and the adherence to an increasing number of lifestyle recommendations. Furthermore, possible correlations among lifestyle behaviors need to be identified to understand possible mutual influences.

As theoretical framework for our search into factors that relate to a healthy lifestyle among cancer survivors, we applied the central thoughts and concepts from social cognitive models: the Reasoned Action Approach, the Attitude-Social influence-Efficacy (ASE) model and its successor the Integrated Model for Behavior Change (I-Change-Model) [24-27]. These models assume that behavior can be predicted by a behavioral intention, which is influenced by proximal factors (social cognitive concepts: attitudes, perceived social influences and selfefficacy expectancies), which in turn can be influenced by more distal factors. In the current study, as distal factors we applied socio-demographic, psychological, and cancer related factors.

In recent years, studies identified correlates of physical activity, however, less is known about the correlates of the other lifestyle behaviors. Regarding physical activity, besides cancer related variables (fatigue, physical side effects), attitude, self-efficacy, social support and intention were important correlates in explaining physical activity in cancer survivors [28, 29]. Additionally, exercise history could be identified as important predictor of exercise adherence. However, for intention, perceived behavior control, age, gender, education, physical fitness and psychological features the findings were inconsistent [30, 31]. Considerably fewer publications described possible correlates of healthy diet, alcohol consumption, and smoking in cancer survivors. Madlensky et al. (2008) identified motivation and self-efficacy as strong predictors of the dietary pattern in breast cancer survivors [32]. Current smoking in cancer survivors was correlated with younger age, lower education and income, and greater alcohol consumption, while quitting after cancer diagnosis was associated with having a smoking related type of cancer [33].

The aims of the present study were 1) to assess the prevalence of lifestyle behaviors and the adherence to recommendations in early cancer survivors, 2) to examine correlations between the different health behaviors and 3) to explore the contribution of sociodemographic, cancer-related, psychological features, social cognitive factors and intention to explain lifestyle behaviors and adherence to recommendations. To our knowledge, this is the first study, exploring the combined contribution of distal factors (enclosing cancer specific socio-demographic and psychological factors), more proximal factors (such as attitude, social support, self-efficacy), and intention, derived from social cognitive models to explain five lifestyle behaviors and adherence to recommendations in early cancer survivors with various types of cancer. 


\section{Methods}

We conducted a cross-sectional survey among early cancer survivors with various types of cancer. This study was approved by the Ethics Review Board on Research (cETO) of the Open University of the Netherlands, Heerlen, The Netherlands. The study was carried out in accordance with the American Psychological Association's Ethics Code and the Declaration of Helsinki, 2013 [34]. No further approval by the Medical Research Ethics Committee (MREC) was necessary, because present study did not fall under the Medical Research Involving Human Subjects Act (WMO).

\section{Study population}

Cancer survivors from Dutch outpatient departments of internal medicine, oncology, and urology were invited to participate. Required sample size of the most extensive multiple regression analysis was $N \geq 160$. Inclusion criteria were: adults ( $>18$ years) diagnosed with and treated for one type of cancer with no sign of recurrence at the last control visit; surgery, chemotherapy and/or radiation therapy as primary treatment, which has been completed at least 6 weeks and up to one year ago. Cancer survivors with severe medical, psychiatric of cognitive problems that would interfere with participation were excluded from the study.

\section{Study procedure}

Eighteen hospitals in the South of the Netherlands were approached for recruitment of participants. Medical staff of eight hospitals agreed and recruited cancer survivors in the period from November 2012 until January 2013. Two recruitment strategies were used: 1) selection of cancer survivors through record review by (research) nurses or 2) personal invitations during outpatient clinic visits with oncologist, urologist, or nurse practitioner. Potentially eligible participants received an information letter, an informed consent form, and a survey booklet. A reminder letter followed 2 weeks later. Cancer survivors, who agreed to participate, were asked to provide written informed consent, to complete the questionnaires and to return these documents to the researchers in an enclosed pre-paid envelope.

\section{Measurements}

All measurements concerned self-report questionnaires.

\section{Lifestyle outcome measures}

Physical activity was assessed using the International Physical Activity Questionnaire Short Form (IPAQ Short) [35-37]; standardized questions from Dutch Measuring Instruments for Research on Smoking and Smoking Cessation were used to measure smoking behavior [38]; Nine items from the Dutch standard questionnaire on nutrition measurements were used to determine vegetable and fruit consumption [39, 40]; alcohol consumption was assessed by using four items from the Dutch standard questionnaire on alcohol consumption [39]. Table 1 provides an overview of these measurements and their properties.

\section{Socio-demographic measures}

Socio-demographic items were measured using standard questions on age, gender, marital status, education level ('low': lower vocational education, medium general secondary education; 'medium': secondary vocational education, higher general secondary education; 'high': higher vocational education, university education), income level ('below average': < €1800 per month; 'average': > €1800 and $<€ 2200$ per month; 'above average': > €2200 per

Table 1 Lifestyle outcome measurements

\begin{tabular}{|c|c|c|c|c|c|}
\hline Behavior & Questionnaire/example question & Categories/scales & Items & $\begin{array}{l}\text { Item- } \\
\text { range }\end{array}$ & $\begin{array}{l}\text { Score- } \\
\text { range }\end{array}$ \\
\hline \multirow[t]{3}{*}{ Physical Activity ${ }^{a}$} & \multirow{3}{*}{$\begin{array}{l}\text { IPAQ Short last } 7 \text { days self- } \\
\text { administered format }\end{array}$} & Walking & 2 & & \multirow{3}{*}{$\begin{array}{l}\text { MET- } \\
\text { min/ } \\
\text { week }\end{array}$} \\
\hline & & Moderate intensive activity & 2 & & \\
\hline & & Vigorous intensive activity & 2 & & \\
\hline \multirow[t]{2}{*}{ Smoking } & "Do you currently smoke?" & Current smoking behavior & 1 & $0-1$ & $0-1$ \\
\hline & "Did you smoke in the past?" & $\begin{array}{l}\text { History of smoking (quit smoking before/ after cancer } \\
\text { diagnosis) }\end{array}$ & 1 & $0-1$ & $0-1$ \\
\hline \multirow[t]{2}{*}{ Alcohol consumption } & $\begin{array}{l}\text { Dutch standard questionnaire on } \\
\text { alcohol consumption }\end{array}$ & $\begin{array}{l}\text { Number of days and glasses of alcohol on weekdays and } \\
\text { weekends }\end{array}$ & 4 & $0-6$ & $0-4$ \\
\hline & & Binge drinking ${ }^{b}$ & 1 & $1-8$ & $0-7$ \\
\hline $\begin{array}{l}\text { Vegetable and fruit } \\
\text { consumption }\end{array}$ & $\begin{array}{l}\text { Dutch standard questionnaire on } \\
\text { nutrition }\end{array}$ & $\begin{array}{l}\text { Number of servings fruit/vegetable (spoons, pieces, glasses) } \\
\text { per day and number of days per week }\end{array}$ & 9 & $1-9$ & $0-7$ \\
\hline
\end{tabular}

Note: IPAQ Short: International Physical Activity Questionnaire Short Form; MET: Metabolic Equivalent of Task

${ }^{a} \geq 600 \mathrm{MET}-\mathrm{min} /$ week corresponds to $\geq$ five days per week performing any combination of walking, moderate or vigorous physical activities

$\mathrm{b} \geq$ Six servings of alcohol during one day

${ }^{c}$ Vegetable consumption was expressed in grams per day. The total score for fruit consumption was the number of servings of fruit per day (up to $100 \mathrm{~g}$ fruit may be replaced by fruit juice) 
month), employment status ('working': self-employed, in paid employment; 'not working': unemployed, retired, unable to work).

\section{Cancer-related measures}

Standard questions were used to assess cancer-related factors. Type of cancer was subsequently categorized into breast, colon, and other types; because of insufficient numbers of the separate types of cancer for appropriate statistical analyses (see footnote Table 3). Type of treatment was categorized into surgery alone, surgery \& chemotherapy, surgery \& radiation, surgery, chemotherapy \& radiation, and other types for the same reason. Aftercare participation was dichotomized (yes/no). Information on length and weight were used to calculate the body mass index (BMI).

\section{Psychological measures}

Table 2 provides an overview of the psychological measures and their properties. Quality of life (QoL) was assessed by using the European Organisation for Research and Treatment of Cancer (EORTC QLQ-C 30) [41-43]. Anxiety and depression were measured by applying the Hospital Anxiety and Depression Scale (HADS) [44-46]. Adjustment to cancer was assessed

Table 2 Psychological outcome measures

\begin{tabular}{|c|c|c|c|c|c|c|}
\hline Concept & Instrument & Subscales used & Items & $\begin{array}{l}\text { Score- } \\
\text { range }\end{array}$ & a & Higher scores indicates \\
\hline \multirow[t]{17}{*}{ Quality of life } & EORTC & Global health status & 2 & $0-100$ & .88 & Better overall health and quality of life \\
\hline & QLQ-C30 & & & & & \\
\hline & & Physical functioning & 5 & $0-100$ & .72 & Better functioning \\
\hline & & Role functioning & 2 & $0-100$ & .86 & Better functioning \\
\hline & & Emotional functioning & 4 & $0-100$ & .86 & Better functioning \\
\hline & & Cognitive functioning & 2 & $0-100$ & .70 & Better functioning \\
\hline & & Social functioning & 2 & $0-100$ & .70 & Better functioning \\
\hline & & Fatigue & 3 & $0-100$ & .87 & Higher level of problems \\
\hline & & Nausea and vomiting & 2 & $0-100$ & .52 & Higher level of problems \\
\hline & & Pain & 2 & $0-100$ & .82 & Higher level of problems \\
\hline & & Dyspnea & 1 & $0-100$ & & Higher level of problems \\
\hline & & Insomnia & 1 & $0-100$ & & Higher level of problems \\
\hline & & Appetite loss & 1 & $0-100$ & & Higher level of problems \\
\hline & & Constipation & 1 & $0-100$ & & Higher level of problems \\
\hline & & Diarrhea & 1 & $0-100$ & & Higher level of problems \\
\hline & & Financial difficulties & 1 & $0-100$ & & Higher level of problems \\
\hline & & & & & & Higher level of problems \\
\hline \multirow{2}{*}{$\begin{array}{l}\text { Anxiety, } \\
\text { depression }\end{array}$} & HADS & Anxiety & 7 & $0-21$ & .84 & More morbidity \\
\hline & & Depression & 7 & $0-21$ & .80 & More morbidity \\
\hline \multirow{7}{*}{$\begin{array}{l}\text { Adjustment to } \\
\text { cancer }\end{array}$} & MAC & Positive adjustment & & & .78 & More positive adjustment \\
\hline & & Fighting spirit & 16 & $16-64$ & & \\
\hline & & Avoidance & 1 & $1-4$ & & \\
\hline & & Negative adjustment & & & .84 & More negative adjustment \\
\hline & & Helplessness/Hopelessness & 6 & $6-24$ & & \\
\hline & & Anxious preoccupation & 9 & $9-36$ & & \\
\hline & & Fatalism & 8 & $8-32$ & & \\
\hline $\begin{array}{l}\text { Illness } \\
\text { perception }\end{array}$ & Brief IPQ & $\begin{array}{l}\text { Consequences, Timeline, Personal control, Identity, } \\
\text { Concern, Coherence, Emotional representation }\end{array}$ & 7 & $0-70$ & .80 & More threatening view of the illness \\
\hline \multirow{2}{*}{$\begin{array}{l}\text { Problem } \\
\text { solving } \\
\text { orientation }\end{array}$} & SPSI-R:S & Positive problem orientation & 5 & $0-4$ & .72 & $\begin{array}{l}\text { Positive outcome and self-efficacy } \\
\text { expectancies, less emotional distress }\end{array}$ \\
\hline & & Negative problem orientation & 5 & $0-4$ & .86 & $\begin{array}{l}\text { Negative outcome and self-efficacy } \\
\text { expectancies, more emotional distress }\end{array}$ \\
\hline
\end{tabular}

Note: QLQ-C30: Quality of Life Questionnaire; HADS: Hospital Anxiety and Depression Scale; MAC: Mental Adjustment to Cancer Scale; Brief IPQ: Brief IIIness Perception Questionnaire; SPSI-R:S: Short Social Problem Solving Inventory-Revised; a: Cronbach's a 
using the Mental Adjustment to Cancer Scale (MAC) [47-49]. Illness perception was assessed with the Brief Illness Perception Questionnaire (Brief IPQ) [50, 51]. The items of the latter questionnaire were adjusted to focus on recovery from cancer, and item 4 (treatment control) was deleted to achieve an acceptable internal consistency (increase Cronbach's alpha from .61 to .75 after removing item 4). Problem solving orientation was measured by using the Short Social Problem Solving Inventory-Revised (SPSI-R:S) [52].

\section{Social cognitive measures}

Attitude, social support, self-efficacy, and intention for each lifestyle behavior were measured by using single items for the separate concepts consisting of 5-point scales with a score ranging from 1 to 5 . Attitude was assessed with questions such as "Is it important for you to follow the nutrition guidelines?" Answer options were yes, very important (5), yes, important (4), not important/not unimportant (3), no, not important (2), no, not at all important (1). Social support was measured by asking questions such as "To what extent do you get support from people who are important to you, to exercise sufficiently?" Response options were always (5), often (4), sometime (3), seldom (2), never (1). Self-efficacy was assessed by asking questions such as "Is it easy or difficult for you to exercise according to the guidelines?" Answering choices were very easy (5), easy (4), not difficult/not easy (3), difficult (2) very difficult (1). Intention was measured by asking questions such as "Do you intend to eat 2 servings of fruit a day in the next 6 months?" Response options were yes, certainly (5), yes, probably (4), maybe/maybe not (3), no, probably not (2), no, certainly not (1). Prior research also applied similar items to measure social cognitive concepts [53-57].

\section{Statistical analyses}

Analyses were conducted using SPSS 21. We used descriptive statistics to describe participant characteristics and the prevalence of health behaviors. For describing the adherence to separate recommendations, we constructed two categories (yes, no) for all five health behaviors.

Missing values were handled according to the questionnaire manuals. For the EORTC QLQ-C30, HADS, and MAC the permitted number of missing values was one. For the SHORT SPSI-R two missing values were permitted. The missing values were supplemented by using mean substitution, as recommended. Cases with missing values on days and time (physical activity), days and number of servings (nutrition and alcohol) were removed from analysis. For other measures, less than $5 \%$ of the values were missing per value in a random pattern. We applied mean substitution for continuous covariates and for categorical covariates, we substituted the values of the modus.

To assess the contribution of the distal and proximal factors in explaining alcohol, vegetable, and fruit consumption, and physical activity we conducted four sequential multiple linear regression analyses [58]. The variables were entered in four entry steps based on the social cognitive models (e.g. Reasoned Action Approach [27], I-Change-Model [26]), the theoretical framework of the present study. The models prescribe an ordering of steps. This implies that socio-demographic and cancerrelated factors were entered in order to control for their possible influence. Then, the psychological factors were entered in step 2 to evaluate what they add to the explanation of variance over and above the first set, the background variables. Subsequently, in step 3, the influence of attitude, social support, and self-efficacy were assessed above the two prior sets. Intention was added it in the last step, according to the assumptions of the social cognitive theories, that intention is influenced by the prior added proximal factors.

To explore the correlates of smoking behavior (smoking vs quitting) among former smokers and current smokers, we conducted sequential logistic regression analysis [58]. Never-smokers were excluded from this analysis. In the logistic regression analysis, we applied the same entry steps as described above. Results from sequential logistic regression analysis $(N$ =139) revealed large confidence intervals, due to the relative small number of participants and a large number of independent variables. Consequently, we conducted a second sequential logistic regression analysis, including fewer variables. The insignificant socio-demographic variables were removed, but core variables were entered in step 1 (age, gender, education level, type of cancer, and type of treatment). Significant psychological variables were added in entry step 2, such as the significant concepts from the EORTC QLQ-C30 (global health/QoL, cognitive functioning, social functioning, nausea /vomiting, insomnia, financial difficulties), and the subscales anxiety and depression from the HADS). In entry step 3 attitude, social support, and self-efficacy were added, and intention was added in the last step.

Furthermore, we were interested in the correlates to explain the overall degree of adherence to lifestyle recommendations. Therefore, we conducted sequential multiple regression analysis and applied the same protocol as described for the multiple regression analyses.

Moreover, correlations between the continuously measured lifestyle behaviors (alcohol, vegetable, fruit consumption, physical activity) were assessed, using Spearman's correlation due to non-normally distributed data. Additionally, by conducting Chi-square tests 
among the five adherence scores we assessed the correlations between adherence to different health behaviors.

\section{Results}

\section{Recruitment and characteristics of the sample}

In total, 455 cancer survivors were invited to participate in the study, 172 (37.8 \%) cancer survivors declined participation, 22 (4.8\%) cancer survivors did not meet the inclusion criteria, and six (1.3\%) respondents did not return the informed consent form. We included 255 $(56 \%)$ respondents in the analysis. Participants' descriptive characteristics are displayed in Table 3. The prevalence of lifestyle behaviors is displayed in Table 4, and the adherence to recommendations is shown in Fig. 1.

\section{Correlations between the different lifestyle behaviors}

We explored mutual correlations between the continuously measured lifestyle behaviors (alcohol, fruit, vegetable consumption, physical activity). Fruit consumption was significantly positively correlated to vegetable consumption, $r_{\mathrm{s}}=.24, p<.001$, and we found a negative relationship between fruit consumption and alcohol consumption $r_{\mathrm{s}}=-.14, p<.05$, which indicated that as fruit consumption was higher, alcohol consumption was lower. No other significant correlations were found.

Furthermore, we explored correlations between adherence (yes, no) to the five different health recommendations and found a statistically significant association between adherence to the smoking and fruit consumption recommendations $\left(\chi^{2}(1)=6.285, p<.05\right)$, however, the effect size represented a low association (Cramer's $V$ $=.16, p<.05)$. Crosstabs showed, that in smokers, $37.8 \%$ met the fruit recommendations, while in non-smokers (former smoker or never-smoker), $58.3 \%$ adhered to the fruit recommendations. No further associations were found between other adherence scores.

\section{Correlates of lifestyle behaviors and adherence to recommendations}

The results of the regression analyses to explain lifestyle behaviors and adherence to recommendations are presented in Table 5 en Table 6.

\section{Alcohol consumption}

Being male $(p=.033)$ and lower self-efficacy toward adherence to the alcohol recommendation $(p=.019)$ were correlated to a higher alcohol consumption. Less problems of insomnia $(p=.058)$ contributed to a lesser extent to a higher alcohol consumption. Before intention was added to the model, higher levels of attitude and lower self-efficacy contributed significantly.

\section{Vegetable consumption}

Significant correlates of a higher vegetable consumption were: 1) a stronger intention toward adhering to the vegetable recommendation $(p=.000), 2)$ higher scores on positive mental adjustment $(p=.022), 3)$ a longer period since completion of primary treatment $(p=.032)$, and, to a smaller extent, lower age $(p=.067)$. A higher attitude and self-efficacy were significantly correlated with vegetable consumption before intention was added to the model.

\section{Fruit consumption}

A stronger intention toward adherence to the fruit recommendation was the only significant correlate in explaining a higher fruit consumption $(\mathrm{p}=.000)$. Before intention was added to the model, lower levels of depressive symptoms, and higher self-efficacy contributed significantly.

\section{Physical activity}

Significant correlates in explaining a higher amount of physical activity were 1$)$ younger ages $(p=.028), 2)$ higher scores on self-efficacy toward adherence to the physical activity recommendation $(p=.005), 2)$ more pain $(p=.039)$, more fatigue $(p=.041)$. Before intention was added to the model, higher levels of attitude and self-efficacy also contributed significantly.

\section{Not smoking}

1) A more positive attitude toward not smoking ( $p$ $=.003), 2)$ higher self-efficacy toward not smoking $(p=.002), 3)$ lower levels of anxiety $(p=.015)$, and 4$)$ better social functioning $(p=.038)$ were significantly correlated to not smoking among (former) smokers. Lower scores on global health/QoL $(p=.052)$, lower scores on cognitive functioning $(p=0.55)$, and not having colon cancer $(p=.053)$ contributed to a smaller extent.

\section{Adherence to lifestyle recommendations}

Significant correlates in explaining adherence to an increasing number of lifestyle recommendations were 1) a more positive intention toward following fruit $(p=.000)$ recommendation, 2) higher scores on self-efficacy toward not smoking $(p=.000)$, a more positive attitude toward following the nutrition recommendations ( $p$ $=.010$ ), and 3 ) three psychological factors (role functioning, $p=.027$; cognitive functioning, $p=.026$; positive mental adjustment to cancer, $p=.045$ ). In addition, a longer period after completing primary cancer treatment $(p=.024)$ and female gender $(p=.39)$ contributed to the adherence to lifestyle recommendations. 
Table 3 Characteristics of the sample $(N=255)$

\begin{tabular}{|c|c|c|c|}
\hline \multicolumn{2}{|l|}{ Variable } & \multicolumn{2}{|l|}{ Variable } \\
\hline Age years (SD) & $60.6(10.7)$ & Type of cancer & \\
\hline Gender & & Breast, n (\%) & $150(58.8)$ \\
\hline Female, n (\%) & $193(70.7)$ & Colon, n (\%) & $51(20)$ \\
\hline Marital status & & Other, n (\%) ${ }^{a}$ & $54(21.1)$ \\
\hline Living with partner, n (\%) & $217(86.5)$ & Type of treatment & \\
\hline Educational level & & Surgery alone, n (\%) & $32(12.6)$ \\
\hline Low, n (\%) & $137(54.6)$ & Surgery and chemotherapy, n (\%) & $55(21.7)$ \\
\hline Medium, n (\%) & $47(18.7)$ & Surgery and radiotherapy, $\mathrm{n}(\%)$ & $46(18.1)$ \\
\hline High, n (\%) & $67(26.3)$ & Surgery, chemo- \& radiotherapy, n (\%) & $92(36.2)$ \\
\hline Employment status & & Other, n (\%) & $29(11.4)$ \\
\hline Not working, n (\%) & $158(64)$ & Participation in aftercare & \\
\hline Income level & & Yes, n (\%) & $134(53)$ \\
\hline Below average, n (\%) & $51(21.1)$ & Number of weeks after treatment, mean (SD) & $26.5(12.7)$ \\
\hline Average, n (\%) & $70(28.9)$ & HADS, mean, (SD) & $8.2(6.7)$ \\
\hline Above average, $n(\%)$ & $121(50)$ & HADS anxiety, mean (SD) & $4.7(3.9)$ \\
\hline BMI, mean (SD) & $26.7(9.4)$ & HADS depression, mean (SD) & $3.5(3.5)$ \\
\hline < 18,5: underweight, $\mathrm{n}(\%)$ & $1(0.4)$ & MAC & \\
\hline 18,5-25: healthy weight, n (\%) & $113(45.7)$ & Positive adjustment, mean (SD) & $51.1(7.0)$ \\
\hline 25-30: overweight, n (\%) & $95(38.5)$ & Negative adjustment, mean (SD) & $29.6(7.0)$ \\
\hline 30-35: obesity, n (\%) & $25(10.1)$ & $\mathrm{IPQR}$, mean (SD) & $32.5(10.9)$ \\
\hline > 35: extreme obesity, n (\%) & $13(5.3)$ & SPSIR & \\
\hline EORTC QLQ-C30 & & Positive problem orientation, mean (SD) & $2.4(0.8)$ \\
\hline Global health status, mean (SD) & $78.1(16.5)$ & Negative problem orientation, mean (SD) & $1.1(0.9)$ \\
\hline Physical functioning, mean (SD) & $85(15.3)$ & Alcohol Attitude, mean (SD) & $2.2(1.3)$ \\
\hline Role functioning, mean (SD) & $79.4(23.8)$ & Social support, mean (SD) & $2.2(1.5)$ \\
\hline Emotional functioning, mean (SD) & $80.1(20.4)$ & Self-efficacy, mean (SD) & $3.6(1.3)$ \\
\hline Cognitive functioning, mean (SD) & $80.6(22)$ & Intention, mean (SD) & $2.4(1.5)$ \\
\hline Social functioning, mean (SD) & $82.8(21.4)$ & Physical Activity Attitude, mean (SD) & $4.6(0.5)$ \\
\hline Body Image, mean (SD) & $82.3(22.8)$ & Social support, mean (SD) & $3.6(1.2)$ \\
\hline Fatigue, mean (SD) & $27(23.9)$ & Self-efficacy, mean (SD) & $3.5(1.1)$ \\
\hline Nausea and Vomiting, mean (SD) & $3.3(10.3)$ & Intention, mean (SD) & $4.7(0.7)$ \\
\hline Pain, mean (SD) & $15.9(22.6)$ & Nutrition Attitude, mean (SD) & $4.1(0.7)$ \\
\hline Dyspnea, mean (SD) & $12(21.9)$ & Social support, mean (SD) & $3.1(1.3)$ \\
\hline Insomnia, mean (SD) & $26.1(28)$ & Self-efficacy, mean (SD) & $3(0.9)$ \\
\hline Appetite loss, mean (SD) & $6.2(16.6)$ & Intention vegetable consumption, mean (SD) & $4.2(1.0)$ \\
\hline Constipation, mean (SD) & $8.2(18.4)$ & Intention fruit consumption, mean (SD) & $4.0(1.1)$ \\
\hline Diarrhea, mean (SD) & $7.5(20)$ & & \\
\hline Financial difficulties, mean (SD) & $10.6(22.5)$ & & \\
\hline
\end{tabular}

Notes: $n$ : numbers of participants; SD: standard deviation; BMI: Body Mass Index; EORTC: European Organisation for Research and Treatment of Cancer; QoL: Quality of Life; HADS: Hospital Anxiety and Depression Scale;MAC: Mental Adjustment to Cancer scale; IPQ: Illness Perception Questionnaire; SPSIR-R:S Short Social Problem Solving Inventory-Revised

${ }^{a}$ other types of cancer were prostate (9\%); Non-Hodgkins's lymphpma (5.9 \%), ovarian (3.1 \%); bladder (1.2 \%); cervix (0.4 \%); Hodgkins's lymphpma (0.4 \%) 
Table 4 Lifestyle behaviors of the sample

\begin{tabular}{|c|c|c|c|c|}
\hline \multirow[t]{2}{*}{ Behavior } & \multirow[b]{2}{*}{ Mean (SD) } & \multirow[b]{2}{*}{ Median (IQR) } & \multicolumn{2}{|c|}{ Meet recommendations } \\
\hline & & & Yes, n (\%) & No, n (\%) \\
\hline \multicolumn{5}{|l|}{ Smoking $(n=250)$} \\
\hline Never & & & $108(43.2)$ & \\
\hline Former & & & $97(38.8)$ & \\
\hline Current & & & & $45(18)$ \\
\hline Alcohol consumption $(n=244)^{1}$ & & & $184(75.4)$ & $60(24.6)$ \\
\hline Never & & & $58(22.8 \%)$ & \\
\hline Social $(n=186)$ & & & $126(67.7 \%)$ & \\
\hline Excessive $(n=186)$ & & & & $60(32.3 \%)$ \\
\hline Male drinkers $(n=60)^{1.1}$ & & & $39(65 \%)$ & $21(35 \%)$ \\
\hline Female drinkers $(n=126)^{1.2}$ & & & $87(69 \%)$ & $39(31 \%)$ \\
\hline Vegetable consumption ${ }^{2}(n=248)$ & $167.7(90.8)$ & $150(107.2-203.6)$ & $68(27.4)^{2.1}$ & $180(72.6)$ \\
\hline Fruit consumption ${ }^{3}(n=252)$ & $1.8(1.1)$ & $2(1-2)$ & $138(54.8)^{3.1}$ & $114(45.2)$ \\
\hline \multicolumn{5}{|l|}{ Physical activity in MET-min/week ${ }^{4}$} \\
\hline Walking $(n=234)$ & $1299.3(1188.5)$ & $924(396-2079)$ & & \\
\hline Moderate $(n=232)$ & $1600.6(1623.8)$ & $1200(210-2400)$ & & \\
\hline Vigorous $(n=235)$ & $962.9(1734.5)$ & $0(0-1440)$ & & \\
\hline Total MET-min/week $(n=247)^{4.1}$ & $3657.6(3293.4)$ & $2613(1284-5145)$ & $216(87.4)^{4.2}$ & $31(12.6)$ \\
\hline
\end{tabular}

Notes: $n$ : numbers of participants; SD: standard deviation; IQR: interquartile range; MET: Metabolic Equivalent of Task

${ }^{1}$ number of alcohol consumptions per week; ${ }^{1.1}$ male: $\leq 14$ drinks per week; ${ }^{1.2}$ female: $\leq 7$ drinks per week

${ }^{2}$ vegetable consumption per day in grams; ${ }^{2.1} \geq 200 \mathrm{~g}$ vegetables per week

${ }^{3}$ number of fruit servings (à $100 \mathrm{~g}$ ) a day. Up to $100 \mathrm{~g}$ fruit may be replaced by $150 \mathrm{~g}$ of fruit juice. ${ }^{3.1}$ at least 2 servings of fruit per week

${ }^{4} \mathrm{MET}-\mathrm{min} /$ week = metabolic equivalent ${ }^{*}$ minutes per week; ${ }^{4.1}$ Total MET-min/week = walking + moderate + vigorous; ${ }^{4.2}>600 \mathrm{MET}$ min $\mathrm{p} / \mathrm{week}$

\section{Discussion}

This cross-sectional study assessed the prevalence and correlates of five lifestyle behaviors in early cancer survivors. Additionally, contributing factors to explain the extent of adherence to lifestyle recommendations were assessed, from which only little evidence is available up to date. The special feature of this study is, that for the first time both, distal and proximal factors, derived from social cognitive theories, were assessed. In all analyses, the required number of participants, in terms of power, has been achieved. Valuable information was gained about important factors that may explain engagement in lifestyle behaviors and the extent of adherence to recommendations. The highest prevalence in followed recommendations have been detected in physical activity (87.4 \%), refrain from smoking (82 \%), and alcohol

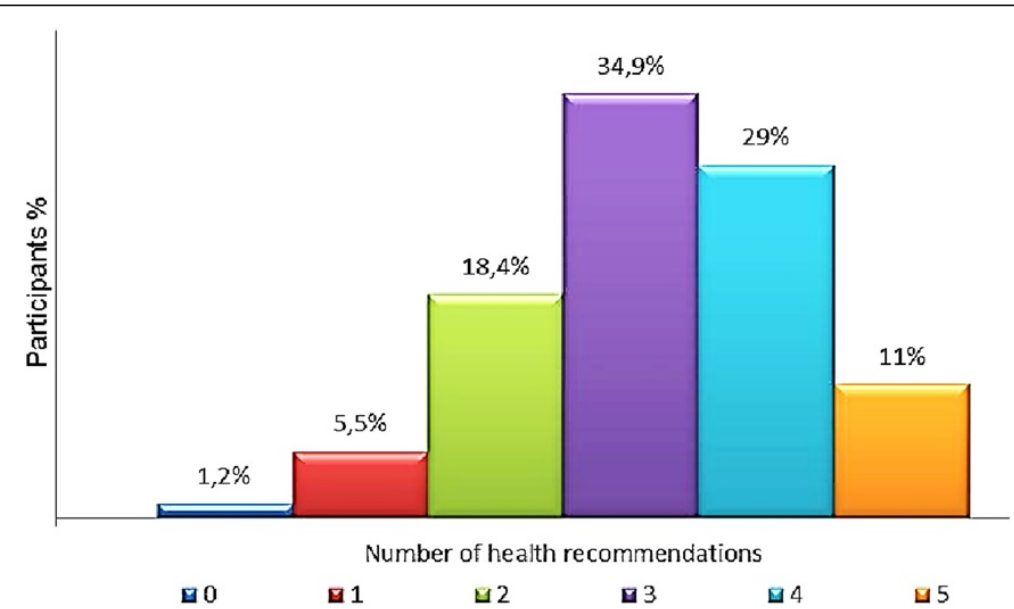

Fig. 1 Adherence to lifestyle recommendations $(N=255)$. Note: The five recommendations relate to physical activity, not smoking, alcohol, fruit and vegetable consumption 
Table 5 Correlates of lifestyle behaviors

\begin{tabular}{|c|c|c|c|c|c|c|c|c|c|c|c|c|c|c|c|}
\hline \multirow[b]{4}{*}{ Variable } & \multicolumn{15}{|c|}{ Lifestyle behavior } \\
\hline & \multirow{2}{*}{\multicolumn{3}{|c|}{$\frac{\text { Number of alcohol consumption }}{(N=223)}$}} & \multirow{2}{*}{\multicolumn{3}{|c|}{$\frac{\text { Number of vegetable consumption }}{(N=225)}$}} & \multirow{2}{*}{\multicolumn{3}{|c|}{$\begin{array}{l}\text { Number of fruit consumption } \\
(N=228)\end{array}$}} & \multirow{2}{*}{\multicolumn{3}{|c|}{$\begin{array}{l}\text { Amount of physical activity } \\
(N=225)\end{array}$}} & \multirow{2}{*}{\multicolumn{3}{|c|}{$\frac{\text { Nonsmoking }}{(N=141)^{\mathrm{a}}}$}} \\
\hline & & & & & & & & & & & & & & & \\
\hline & $\mathrm{B}$ & $(95 \% \mathrm{Cl})$ & $p$ & $B$ & $(95 \% \mathrm{Cl})$ & $p$ & $B$ & $(95 \% \mathrm{Cl})$ & $p$ & $B$ & $(95 \% \mathrm{Cl})$ & $p$ & ExpB & $(95 \% \mathrm{Cl})$ & $p^{c}$ \\
\hline Age & .037 & $(-.15 ; .23)$ & .698 & -1.307 & $(-2.70 ; .09)$ & .067 & .007 & $(-.01 ; .02)$ & .367 & -13.723 & $(-25.94 ;-1.51)$ & $.028^{*}$ & .936 & $(.86 ; 1.02)$ & .127 \\
\hline Female gender & -5.805 & $(-11.13 ;-4.77)$ & $.033^{*}$ & 7.579 & $(-32.98 ; 48.14)$ & .713 & .211 & $(-.20 ; .63)$ & .315 & -11.993 & $(-361.07 ; 337.08)$ & .946 & .394 & $(.04 ; 4.45)$ & .399 \\
\hline \multicolumn{16}{|l|}{ Marital status } \\
\hline Without partner & ref & & & ref & & & ref & & & ref & & & & & \\
\hline With partner & -4.986 & $(-10.75 ; .73)$ & .089 & 24.032 & $(-17.65 ; 65.72)$ & .257 & .174 & $(-.25 ; .60)$ & .421 & -122.543 & $(-478.92 ; 233.83)$ & .498 & & & \\
\hline \multicolumn{16}{|l|}{ Education } \\
\hline Low & ref & & & ref & & & ref & & & ref & & & ref & & .198 \\
\hline Medium & -1.165 & $(-5.60 ; 3.72)$ & .605 & 14.521 & $(-17.78 ; 64.82)$ & .376 & -.019 & $(-.35 ; .31)$ & .907 & -176.621 & $(-451.64 ; 98.40)$ & .207 & 2.664 & $(.52 ; 13.75)$ & .242 \\
\hline High & -2.370 & $(-6.64 ; 1.90)$ & .274 & 18.004 & $(-1.71 ; 49.72)$ & .264 & .075 & $(-.25 ; .40)$ & .644 & -215.435 & $(-474.80 ; 43.93)$ & .103 & 5.451 & $(.72 ; 41.44)$ & .101 \\
\hline \multicolumn{16}{|l|}{ Income } \\
\hline Above average & ref & & & ref & & & ref & & & ref & & & & & \\
\hline Average & -2.646 & $(-6.42 ; 1.13)$ & .168 & -19.041 & $(-47.66 ; 9.58)$ & .191 & -.118 & $(-.41 ; .17)$ & .423 & 71.279 & $(-169.63 ; 312.19)$ & .560 & & & \\
\hline Below average & -4.183 & $(-9.78 ; 1.41)$ & .142 & -6.040 & $(-47.44 ; 35.36)$ & .774 & .022 & $(-.40 ; .45)$ & .917 & -77.931 & $(-431.78 ; 275.19)$ & .654 & & & \\
\hline \multicolumn{16}{|l|}{ Cancer type } \\
\hline Other & ref & & & ref & & & ref & & & ref & & & ref & & .096 \\
\hline Breast & -1.244 & $(-7.97 ; 5.48)$ & .716 & -1.055 & $(-51.79 ; 49.68)$ & .967 & -.046 & $(-.56 ; .47)$ & .862 & 137.614 & $(-291.14 ; 566.37)$ & .527 & .489 & $(.02 ; 12.11)$ & .662 \\
\hline Colon & -1.859 & $(-8.18 ; 4.46)$ & .562 & 5.333 & $(-42.80 ; 53.47)$ & .827 & .002 & $(-.49 ; .49)$ & .995 & 15.656 & $(-390.56 ; 421.88)$ & .939 & .045 & $(.00 ; 1.04)$ & .053 \\
\hline \multicolumn{16}{|l|}{ Treatment } \\
\hline$A \|^{\mathrm{b}}$ & ref & & & ref & & & ref & & & ref & & & ref & & .554 \\
\hline Surgery alone & 1.914 & $(-4.18 ; 8.01)$ & .536 & -6.261 & $(-51.72 ; 39.20)$ & .786 & -.077 & $(-.54 ; .39)$ & .745 & -67.186 & $(-443.93 ; 309.56)$ & .725 & .483 & $(.04 ; 5.73)$ & .565 \\
\hline Surgery, chemo & .991 & $(-3.40 ; 5.38)$ & .657 & -.259 & $(-33.72 ; 33.20)$ & .988 & -0.52 & $(-.39 ; .29)$ & .764 & 56.566 & $(-223.74 ; 3236.87)$ & .691 & 3.975 & $(.38 ; 41.21)$ & .247 \\
\hline Surgery, radiation & .228 & $(-4.88 ; 5.34)$ & .930 & -15.436 & $(-52.36 ; 21.49)$ & .411 & .031 & $(-.35 ; .21)$ & .872 & -43.457 & $(-358.83 ; 271.92)$ & .786 & .471 & $(.06 ; 3.68)$ & .473 \\
\hline Other & -.041 & $(-7.96 ; 7.88)$ & .992 & -.947 & $(-60.33 ; 58.44)$ & .975 & -.144 & $(-.74 ; .45)$ & .633 & -70.914 & $(-559.88 ; 418.05)$ & .775 & 1.275 & $(.04 ; 43.16)$ & .893 \\
\hline \multicolumn{16}{|l|}{ Aftercare } \\
\hline No & ref & & & ref & & & ref & & & ref & & & & & \\
\hline Yes & 1.914 & $(-5.00 ; 2.39)$ & .487 & -15.766 & $(-43.27 ; 11.74)$ & .260 & -.070 & $(-.35 ; .21)$ & .622 & -60.426 & $(-291.29 ; 170.44)$ & .606 & & & \\
\hline Time after treatment & .991 & $(-.236 ; .026)$ & .117 & 1.053 & $(.09 ; 2.02)$ & $.032^{*}$ & .003 & $(-.01 ; .01)$ & .500 & .787 & $(-7.26 ; 8.83)$ & .874 & & & \\
\hline BMI & -.257 & $(-.654 ; .14)$ & .203 & 1.654 & $(-1.32 ; 4.63)$ & .274 & -.013 & $(-.04 ; .02)$ & .376 & -7.844 & $(-33.21 ; 17.53)$ & .453 & & & \\
\hline Glob. Health/ QoL & .044 & $(-.10 ; .12)$ & .543 & -.412 & $(-1.48 ; .67)$ & .451 & -.002 & $(-.01 ; .01)$ & .732 & .246 & $(-8.74 ; 9.23)$ & .957 & .926 & $(.86 ; 1.00)$ & .052 \\
\hline Physical funct. & .008 & $(-.17 ; .18)$ & .929 & -.676 & $(-1.99 ; .46)$ & .313 & -.007 & $(-.02 ; .01)$ & .314 & 5.129 & $(-6.24 ; 16.50)$ & .374 & & & \\
\hline
\end{tabular}


Table 5 Correlates of lifestyle behaviors (Continued)

\begin{tabular}{|c|c|c|c|c|c|c|c|c|c|c|c|c|c|c|c|}
\hline Role funct. & -.076 & $(-.20 ; .05)$ & .199 & .521 & $(-.38 ; 1.42)$ & .254 & .006 & $(-.00 ; .02)$ & .164 & 4.379 & $(-3.05 ; 11.81)$ & .246 & & & \\
\hline Emotional funct. & -.078 & $(-.20: .05)$ & .226 & .263 & $(-.71 ; 1.24)$ & .593 & .003 & $(-.01 ; .01)$ & .554 & 7.327 & $(-.94 ; 15.59)$ & .082 & & & \\
\hline Cognitive funct. & -.013 & $(-.12 ; .09)$ & .796 & -.079 & $(-.86 ; .70)$ & .840 & -.002 & $(-.01 ; .01)$ & .600 & .192 & $(-7.84 ; 6.51)$ & .953 & .957 & $(.92 ; 1.00)$ & .055 \\
\hline Social funct. & .070 & $(-.05 ; .19)$ & .234 & -.466 & $(-1.32 ; .39)$ & .285 & -.003 & $(-.01 ; .01)$ & .488 & -.661 & $(-7.84 ; 6.51)$ & .856 & 1.046 & $(1.00 ; 1.09)$ & $.038^{*}$ \\
\hline Body Image & .016 & $(-.07 ; .10)$ & .703 & .010 & $(-.65 ; .66)$ & .977 & .000 & $(-.01 ; .01)$ & .935 & 1.977 & $(-3.43 ; 7.38)$ & .471 & & & \\
\hline Fatigue & .046 & $(-.07 ; .16)$ & .436 & .269 & $(-.63 ; 1.16)$ & .554 & .001 & $(-.01 ; .01)$ & .744 & 7.732 & $(.30 ; 12.15)$ & $.041^{*}$ & & & \\
\hline Nausea,vomiting & -.043 & $(-.23 ; .15)$ & .651 & -.071 & $(-1.56 ; 1.42)$ & .925 & .000 & $(-.02 ; .01)$ & .957 & -2.743 & $(-14.78 ; 9.29)$ & .654 & .945 & $(.89 ; 1.01)$ & .081 \\
\hline Pain & -.034 & $(-.13 ; .06)$ & .472 & .169 & $(-.56 ; .90)$ & .650 & .001 & $(-.01 ; .01)$ & .784 & 6.229 & $(.31 ; 15.16)$ & $.039^{*}$ & & & \\
\hline Dyspnea & -.025 & $(-.11 ; .06)$ & .553 & -.428 & $(-1.07 ; .21)$ & .188 & -.004 & $(-.01 ; .00)$ & .277 & .010 & $(-5.19 ; 5.21)$ & .997 & & & \\
\hline Insomnia & -.062 & $(-.13 ; .00)$ & .058 & .214 & $(-.27 ; .70)$ & .382 & .000 & $(-.00 ; .01)$ & .866 & .850 & $(-3.13 ; 4.83)$ & .674 & .977 & $(.95 ; 1.01)$ & .108 \\
\hline Appetite loss & .033 & $(-.08 ; .15)$ & .576 & -.168 & $(-1.05 ; .72)$ & .708 & .001 & $(-.01 ; .01)$ & .805 & 4.505 & $(-2.91 ; 11.91)$ & .223 & & & \\
\hline Constipation & -.036 & $(-.12 ; .03)$ & .452 & -.374 & $(-1.10 ; .35)$ & .310 & -.005 & $(-.01 ; .00)$ & .186 & -5.298 & $(-11.12 ; .53)$ & .074 & & & \\
\hline Diarrhea & .030 & $(-.13 ; .06)$ & .503 & .111 & $(-.59 ; .81)$ & .754 & .003 & $(-.01 ; .01)$ & .383 & .151 & $(-5.57 ; 5.87)$ & .959 & & & \\
\hline Financial probl. & .001 & $(-.09 ; .09)$ & .987 & -.339 & $(-.99 ; .31)$ & .302 & -.001 & $(-.01 ; .01)$ & .827 & .024 & $(-5.44 ; 5.49)$ & .993 & .977 & $(.95 ; 1.01)$ & .157 \\
\hline Anxiety & -.080 & $(-.78 ; .62)$ & .820 & -.084 & $(-5.43 ; 5.26)$ & .975 & -.008 & $(-.06 ; .05)$ & .760 & 41.203 & $(-3.24 ; 85.65)$ & .069 & .682 & $(.50 ; .93)$ & $.015^{*}$ \\
\hline Depression & .170 & $(-.60 ; .94)$ & .662 & -.204 & $(-6.03 ; 5.62)$ & .945 & -.027 & $(-.09 ; .03)$ & .365 & -33.248 & $(-80.72 ; 14.22)$ & .169 & 1.187 & $(.89 ; 1,58)$ & .236 \\
\hline Pos. adjustment & -.057 & $(-.32 ; .20)$ & .667 & 2.297 & $(.34 ; 4.26)$ & $.022^{*}$ & .013 & $(-.02 ; .03)$ & .198 & 12.872 & $(-3.001 ; 28.75)$ & .111 & & & \\
\hline Neg. adjustment & -.149 & $(-.48 ; .18)$ & .373 & -1.992 & $(-4.40 ; .42)$ & .105 & .005 & $(-.02 ; .03)$ & .714 & 6.782 & $(-13.48 ; 27.04)$ & .510 & & & \\
\hline Illness perception & -.034 & $(-.24 ; .17)$ & .738 & .782 & $(-.71 ; 2.28)$ & .303 & .010 & $(-.01 ; .03)$ & .189 & -4.296 & $(-16.86 ; 8.27)$ & .501 & & & \\
\hline PPO & 1.324 & $(-1.05 ; 3.70)$ & .273 & -4.790 & $(-22.60 ; 13.02)$ & .596 & -.001 & $(-.18 ; .18)$ & .991 & -78.106 & $(-225.46 ; 69.24)$ & .297 & & & \\
\hline NPO & .457 & $(-1.75 ; 2.66)$ & .684 & 2.240 & $(-14.26 ; 18.74)$ & .789 & -.078 & $(-.25 ; .09)$ & .360 & -82.287 & $(-222.29 ; 57.73)$ & .248 & & & \\
\hline Attitude & 1.522 & $(-.32 ; 3.36)$ & .105 & 17.076 & $(-1.98 ; 36.13)$ & .079 & -.016 & $(-.21 ; .18)$ & .872 & 192.876 & $(-19.74 ; 405.49)$ & .075 & 5.707 & $(1.83 ; 17.7)$ & $.003^{* *}$ \\
\hline Social support & -.004 & $(-.01 ; .00)$ & .290 & -.012 & $(-.19 ; .17)$ & .894 & 0.00 & $(-.00 ; .00)$ & .710 & -.111 & $(-1.62 ; 1.40)$ & .884 & & & \\
\hline Self-efficacy & -1.532 & $(-2.81 ;-.25)$ & $.019^{*}$ & 11.342 & $(-3.70 ; 26.36)$ & .138 & .070 & $(-.09 ; .23)$ & .378 & 181.637 & $(54.71 ; 308.56)$ & $.005^{*}$ & 2.583 & $(1.42 ; 4.69)$ & $.002^{* *}$ \\
\hline Intention & .479 & $(-1.02 ; 1.98)$ & .530 & 36.980 & $(23.16 ; 50.81)$ & $.000^{* *}$ & .597 & $(.48 ; .72)$ & $.000^{* *}$ & 137.551 & $(-23.15 ; 298.25)$ & .093 & .583 & $(.58 ; 1.56)$ & .852 \\
\hline Constant B (SE) & \multicolumn{3}{|c|}{$35.146(20.40)$} & \multicolumn{3}{|c|}{$-105.53(151.51)$} & \multicolumn{3}{|c|}{$-1.63(1.54)$} & \multicolumn{2}{|c|}{$-2456.07(1356.25)$} & & \multicolumn{2}{|c|}{$2.96(5.07)$} & \\
\hline$R^{2}$ & \multicolumn{3}{|l|}{.297} & \multicolumn{3}{|l|}{.415} & \multicolumn{3}{|l|}{.514} & \multicolumn{3}{|l|}{.312} & & & \\
\hline Sig. F Change & \multirow{2}{*}{\multicolumn{3}{|c|}{.530}} & \multicolumn{3}{|l|}{.000} & \multicolumn{3}{|l|}{.000} & \multicolumn{3}{|l|}{.093} & & & \\
\hline Cox \& Snell $R^{2}$ & & & & & & & & & & & & & .518 & & \\
\hline
\end{tabular}


Table 5 Correlates of lifestyle behaviors (Continued)

Nagelkerke $R^{2}$

Model $X^{2}$

102.87

$p$

.000

Note: From Sequential Multiple Regression (continuous outcomes) and Sequential Logistic Regression (smoking) entry step 4 is displayed. Forced entry (enter) method was used; Abbreviations: ExpB: odds ratio; ref:

reference group; BMI: Body Mass Index; EORTC: European Organisation for Research and Treatment of Cancer; QoL: Quality of Life; HADS: Hospital Anxiety and Depression Scale; neg./pos. adjustment from MAC: Menta Adjustment to Cancer Scale; IPQ: Illness Perception Questionnaire; SPSIR-R:S Short Social Problem Solving Inventory-Revised; Chemo: chemotherapy; PPO: positive problem orientation; NPO: negative problem orientation

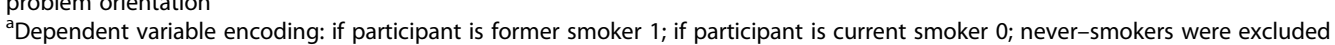

ball = surgery + chemotherapy + radiation

${ }^{c} p$-value of Wald test is presented

${ }^{*} p<0.05 ;{ }^{* *} p<0.01$ 
Table 6 Correlates of adherence to recommendations $(N=236)$

\begin{tabular}{|c|c|c|c|c|c|c|c|c|c|c|c|c|}
\hline \multirow[b]{3}{*}{ Variable } & \multicolumn{12}{|c|}{ Adherence to an increasing number of lifestyle recommendations } \\
\hline & \multicolumn{3}{|c|}{ Model 1} & \multicolumn{3}{|c|}{ Model 2} & \multicolumn{3}{|c|}{ Model 3} & \multicolumn{3}{|c|}{ Model 4} \\
\hline & $\mathrm{B}$ & $(95 \% \mathrm{Cl})$ & $p$ & $\mathrm{~B}$ & $(95 \% \mathrm{Cl})$ & $p$ & $\mathrm{~B}$ & $(95 \% \mathrm{Cl})$ & $p$ & B & $(95 \% \mathrm{Cl})$ & $p$ \\
\hline Age & .000 & $(-.02 ; .02)$ & .962 & .004 & $(-.13 ; .02)$ & .610 & -.006 & $(-.02 ; .01)$ & .441 & -.010 & $(-.02 ; .01)$ & .201 \\
\hline Female gender & .655 & $(.18 ; 1.13)$ & .007 & .686 & $(.18 ; 1.19)$ & .008 & .398 & $(-.60 ; .86)$ & .088 & .462 & $(.02 ; .90)$ & $.039 *$ \\
\hline Marital status: with partner & .566 & $(.08 ; 1.06)$ & .024 & .460 & $(-.55 ; .98)$ & .080 & .284 & $(-.17 ; .74)$ & .221 & .391 & $(-.44 ; .83)$ & .078 \\
\hline \multicolumn{13}{|l|}{ Education, low $=$ ref } \\
\hline Medium & .200 & $(-.19 ; .59)$ & .307 & .215 & $(-.19 ; .62)$ & .291 & .035 & $(-.33 ; .40)$ & .850 & -.019 & $(-.37 ; .33)$ & .915 \\
\hline High & .601 & $(.24 ; .96)$ & .001 & .534 & $(.15 ; .92)$ & .006 & .029 & $(-.34 ; .40)$ & .877 & .117 & $(-.33 ; .47)$ & .517 \\
\hline \multicolumn{13}{|l|}{ Income, above average = ref. } \\
\hline Average & .067 & $(-.28 ; .42)$ & .703 & -.021 & $(-.38 ; .33)$ & .907 & -.063 & $(-.38 ; .25)$ & 695 & -.030 & $(-.33 ; .27)$ & .844 \\
\hline Below average & .062 & $(-.40 ; .52)$ & .789 & .037 & $(-.48 ; .56)$ & .889 & -.203 & $(-.66 ; .26)$ & .384 & -.083 & $(-.52 ; .36)$ & .709 \\
\hline \multicolumn{13}{|l|}{ Cancer type, other $=$ ref } \\
\hline Breast & .459 & $(-.12 ; 1.04)$ & .122 & .281 & $(-.35 ; .91)$ & .381 & .130 & $(-.43 ; .69)$ & .646 & .046 & $(-.47 ; .60)$ & .813 \\
\hline Colon & .291 & $(-.26 ; .84)$ & .296 & .224 & $(-.37 ; 82)$ & .461 & .087 & $(-.41 ; .62)$ & .744 & .145 & $(-.36 ; .65)$ & .570 \\
\hline \multicolumn{13}{|l|}{ 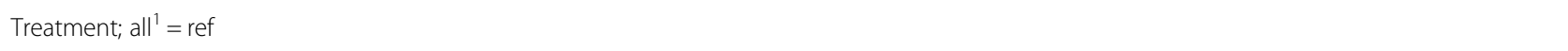 } \\
\hline Surgery alone & .076 & $(-.47 ; .60)$ & .805 & -.004 & $(-.57 ; .56)$ & .988 & .128 & $(-.39 ; .64)$ & .626 & .033 & $(-.46 ; .52)$ & .894 \\
\hline Surgery + chemo & .049 & $(-.31 ; .50)$ & .644 & .053 & $(-.37 ; .47)$ & .803 & .025 & $(-.35 ; .40)$ & .897 & .027 & $(-.33 ; .39)$ & .882 \\
\hline Surgery + radiation & -.467 & $(-.90 ;-.04)$ & .034 & -.459 & $(-.91 ;-.01)$ & .046 & -.209 & $(-.69 ; .11)$ & .157 & -.264 & $(-.66 ; .14)$ & .195 \\
\hline Other & .577 & $(-.11 ; 1.26)$ & .100 & .573 & $(-.48 ; 1.44)$ & .123 & .123 & $(-.53 ; 78)$ & .711 & .107 & $(-.52 ; .73)$ & .736 \\
\hline Participating in aftercare & -.083 & $(-.41 ; .24)$ & .611 & .006 & $(-.33 ; .35)$ & .971 & -.123 & $(-.42 ; .18)$ & .420 & -.105 & $(-.39 ; .18)$ & .472 \\
\hline Time after treatment & .009 & $(-.00 ; .02)$ & .098 & .010 & $(-.01 ; .02)$ & .109 & .009 & $(-.00 ; .02)$ & .091 & .012 & $(.00 ; .02)$ & $.024^{*}$ \\
\hline BMI & .021 & $(-.01 ; .06)$ & .230 & .017 & $(-.02 ; .05)$ & .347 & .013 & $(-.02 ; .05)$ & .445 & .006 & $(-.03 ; .04)$ & .734 \\
\hline Glob. Health/QoL & - & - & - & -.012 & $(-.03 ; .01)$ & .080 & -.010 & $(-.02 ; .00)$ & .086 & -.009 & $(-.02 ; .00)$ & .115 \\
\hline Physical funct. & - & - & - & -.003 & $(-.02 ; .02)$ & .740 & -.004 & $(-.02 ; .01)$ & .604 & -.004 & $(-.02 ; .01)$ & .619 \\
\hline Role funct. & - & - & - & .014 & $(.03 ; .03)$ & .015 & .010 & $(.01 ; 02)$ & .036 & .010 & $(.00 ; .02)$ & $.027^{*}$ \\
\hline Emotional funct. & - & - & - & .001 & $(-.01 ; .01)$ & .854 & .006 & $(-.01 ; .02)$ & .320 & .007 & $(-.00 ; .02)$ & .184 \\
\hline Cognitive funct. & - & - & - & -.012 & $(-.02 ;-.00)$ & .008 & -.009 & $(-.02 ;-.00)$ & .030 & -.009 & $(-.02 ;-.00)$ & $.026^{*}$ \\
\hline Social funct. & - & - & - & -.004 & $(-.01 ; .01)$ & .493 & -.004 & $(-.01 ; .01)$ & .364 & -.004 & $(-.01 ; .01)$ & .400 \\
\hline Body Image & - & - & - & .002 & $(-.01 ; .01)$ & .658 & .002 & $(-.01 ; .01)$ & .657 & .002 & $(-.01 ; .01)$ & .554 \\
\hline Fatigue & - & - & - & .001 & $(-.01 ; .01)$ & .922 & .000 & $(-.01 ; .01)$ & .980 & .001 & $(-.01 ; .01)$ & .779 \\
\hline Nausea en Vomiting & - & - & - & -.010 & $(-.03 ; .01)$ & .254 & -.010 & $(-.03 ; .01)$ & .198 & -.005 & $(-.02 ; .01)$ & .517 \\
\hline Pain & - & - & - & .002 & $(-.01 ; .01)$ & .713 & .005 & $(-.00 ; .01)$ & .243 & .006 & $(-.00 ; .01)$ & .140 \\
\hline Dyspnea & - & - & - & -.006 & $(-.01 ; .00)$ & .124 & .002 & $(-.01 ; .01)$ & .620 & .001 & $(-.01 ; .01)$ & .799 \\
\hline Insomnia & - & - & - & -.002 & $(-.01 ; .00)$ & .576 & .001 & $(-.01 ; .01)$ & .998 & .001 & $(-.01 ; .01)$ & .992 \\
\hline Appetite loss & - & - & - & -.002 & $(-.01 ; .01)$ & .759 & -.001 & $(-.01 ; .01)$ & .856 & -.001 & $(-.01 ; .01)$ & .869 \\
\hline Constipation & - & - & - & -.006 & $(-.01 ; .00)$ & .202 & -.002 & $(-.01 ; .01)$ & .653 & -.002 & $(-.01 ; .00)$ & .544 \\
\hline Diarrhea & - & - & - & -.001 & $(-.01 ; .01)$ & .811 & .001 & $(-.01 ; .01)$ & .862 & -.001 & $(-.01 ; .01)$ & .824 \\
\hline Financial difficulties & - & - & - & -.005 & $(-.01 ; .00)$ & .268 & -.003 & $(-.01 ; .01)$ & .983 & -.002 & $(-.01 ; .01)$ & .531 \\
\hline Anxiety & - & - & - & -.033 & $(-.10 ; .03)$ & .325 & -.007 & $(-.07 ; .05)$ & .818 & -.018 & $(-.07 ; .04)$ & .531 \\
\hline Depression & - & - & - & -.016 & $(-.09 ; .05)$ & .652 & -.049 & $(-.11 ; .01)$ & .127 & -.024 & $(-.09 ; .04)$ & .445 \\
\hline Positive adjustment & - & - & - & .023 & $(.00 ; .05)$ & .048 & .025 & $(.01 ; .05)$ & .016 & .020 & $(.00 ; .04)$ & $.045^{*}$ \\
\hline Negative adjustment & - & - & - & .007 & $(-.02 ; .05)$ & .648 & .026 & $(-01 ; .05)$ & .058 & .023 & $(-.00 ; .05)$ & .095 \\
\hline Illness perception & - & - & - & .005 & $(-.01 ; .02)$ & .573 & .003 & $(-.01 ; .02)$ & .693 & .002 & $(-.01 ; .02)$ & .825 \\
\hline PPO & - & - & - & .042 & $(-.16 ; .24)$ & .684 & -.003 & $(-.19 ; .018)$ & .975 & .006 & $(-.17 ; .18)$ & .944 \\
\hline
\end{tabular}


Table 6 Correlates of adherence to recommendations $(N=236)$ (Continued)

\begin{tabular}{|c|c|c|c|c|c|c|c|c|c|c|c|c|}
\hline NPO & - & - & - & .088 & $(-.12 ; .29)$ & .394 & .115 & $(-.07 ; .30)$ & .216 & .089 & $(-.09 ; .26)$ & .317 \\
\hline Alcohol: Attitude & - & - & - & - & - & - & .084 & $(-.03 ; .20)$ & .158 & .020 & $(-.13 ; .17)$ & .783 \\
\hline Social support & - & - & - & - & - & - & .000 & $(.00 ; .00)$ & .184 & .000 & $(.00 ; .001)$ & .266 \\
\hline Self-efficacy & - & - & - & - & - & - & .052 & $(-.06 ; .16)$ & .344 & .053 & $(-.05 ; .16)$ & .306 \\
\hline Nutrition: Attitude & - & - & - & - & - & - & .373 & $(.17 ; .58)$ & .000 & .265 & $(.06 ; .47)$ & $.010^{*}$ \\
\hline Social support & - & - & - & - & - & - & -.001 & $(-.00 ; .00)$ & .301 & -.001 & $(-.00 ; .00)$ & .295 \\
\hline Self-efficacy & - & - & - & - & - & - & .112 & $(-.06 ; .28)$ & .200 & -.037 & $(-.21 ; .14)$ & .671 \\
\hline Physical Activity: Attitude & - & - & - & - & - & - & -.184 & $(-.46 ; .09)$ & .193 & -.157 & $(-.44 ; .12)$ & .269 \\
\hline Social support & - & - & - & - & - & - & -.001 & $(-.00 ; .00)$ & .170 & -.001 & $(-.00 ; .00)$ & .206 \\
\hline Self-efficacy & - & - & - & - & - & - & .104 & $(-.07 ; .27)$ & .227 & .080 & $(-.09 ; .25)$ & .347 \\
\hline Smoking: Attitude & & & & & & & .153 & $(-.07 ; .38)$ & .184 & .100 & $(-.13 ; .34)$ & .387 \\
\hline Social support & - & - & - & - & - & - & .020 & $(-.06 ; .10)$ & .623 & .000 & $(-.08 ; .08)$ & .993 \\
\hline Self-efficacy & - & - & - & - & - & - & .323 & $(.17 ; .47)$ & .000 & .330 & $(.19 ; 48)$ & $.000^{* *}$ \\
\hline Intention & & & & & & & & & & & & \\
\hline Alcohol cons. & - & - & - & - & - & - & - & - & - & .066 & $(-.05 ; .18)$ & .373 \\
\hline Vegetable cons. & - & - & - & - & - & - & - & - & - & .112 & $(-.04 ; .26)$ & .144 \\
\hline Fruit cons. & - & - & - & - & - & - & - & - & - & .263 & $(.13 ; .39)$ & $.000^{* *}$ \\
\hline Physical activity & - & - & - & - & - & - & - & - & - & .045 & $(-.16 ; .25)$ & .668 \\
\hline Smoking & - & - & - & - & - & - & - & - & - & -.009 & $(-.12 ; .10)$ & .874 \\
\hline Constant B (SE) & \multicolumn{3}{|c|}{$.932(.77)$} & \multicolumn{3}{|c|}{.795 (1.85) } & \multicolumn{3}{|c|}{$-2.728(1.78)$} & \multicolumn{3}{|c|}{$-3.72(1.72)$} \\
\hline$R^{2}$ & \multicolumn{3}{|c|}{.172} & \multicolumn{3}{|l|}{.289} & \multicolumn{3}{|l|}{.502} & \multicolumn{3}{|l|}{.567} \\
\hline Sig. F Change & \multicolumn{3}{|c|}{.000} & \multicolumn{3}{|l|}{.109} & \multicolumn{3}{|l|}{.000} & \multicolumn{3}{|l|}{.000} \\
\hline
\end{tabular}

Note: From Sequential Multiple Regression (continuous outcomes) and Sequential Logistic Regression (smoking) entry step 4 is displayed. Forced entry (enter) method was used. Abbreviations: ExpB: odds ratio; ref: reference group; BMI: Body Mass Index; EORTC: European Organisation for Research and Treatment of Cancer; QoL: Quality of Life; HADS: Hospital Anxiety and Depression Scale; MAC: Mental Adjustment to Cancer Scale; IPQ: IIIness Perception Questionnaire; SPSIRR:S Short Social Problem Solving Inventory-Revised; Chemo: chemotherapy; PPO: positive problem orientation; NPO: negative problem orientation; $\mathrm{R}^{2}$ :correlation coefficient squared

'all = surgery + chemotherapy + radiation

${ }^{2}$ Dependent variable encoding: if participant is former smoker 1 ; if participant is current smoker $0 p$-value of Wald test is presented ${ }^{*} p<0.05$; ${ }^{* *} p<0.01$

consumption (75.4\%). Low prevalence was found in adherence to the fruit recommendation $(54.8 \%)$ and, in particular in adherence to the vegetable recommendation $(27.4 \%)$.

\section{Physical activity}

The proportion of participants meeting the physical activity recommendations $(87.4 \%)$ were much higher than results earlier reported $[1,16,59]$. In these studies, however, a different measurement instrument was used, which might explain the discrepancy. Our results are rather consistent with results from studies, which also used the IPAQ Short form; however, over-reporting might have been occurred [35, 60,61]. An additional explanation for the fairly high level of physical activity might be the relatively good health of the participants. The sample characteristics (Table 1) showed rather high scores on the functioning scales as well as low scores on the symptom scales of the EORTC QLQ-C30, and low scores on the HADS. In addition, more than half of the sample used some form of cancer aftercare, which often has a strong emphasis on physical activity. From the individuals who were engaged in aftercare, almost $50 \%$ were supported by an oncology physiotherapist or participated in a rehabilitation program including physical exercises. This might also partly explain the high level of PA among our sample of survivors.

Higher scores on self-efficacy lower ages, and, more pain, and more fatigue were the only significant correlates of a higher level of physical activity. Causal directions cannot be determined, but a possible explanation for the positive relationships between pain respectively fatigue and a higher level of physical activity could be, that pain and fatigue might have been reasons to get supervised by an (oncological) physiotherapist, or to follow a rehabilitation program. In the Netherlands, guidelines to cope with pain and fatigue are characterized by an active approach (gradually building up physical activity). 
As described before, physical activity is an important modifiable lifestyle behavior, which can have an impact on health outcomes in cancer survivors. Even though most of the cancer survivors meet the recommendations in our study, in clinical practice, attention should be given to the maintenance and if possible, to a gradual increase of physical activity.

\section{Smoking}

Of our sample, $18 \%$ were current smoker, which is a higher rate of smokers compared to findings from other research [33, 62, 63]. Most of the former smokers quitted before cancer diagnosis, and half of the current smokers intended to quit within six months. The strongest correlates of not smoking were a higher selfefficacy, a more positive attitude toward nonsmoking, lower anxiety and better social functioning, while in other research, where social cognitive and psychological variables were not considered, younger age, lower education/ income, greater alcohol consumption, and cancer type were correlated with current smoking [33]. However, qualitative results of Berg et al. [64], confirmed that a positive attitude towards quitting may help to (remain) quit, and that feelings of anxiety and low self-efficacy were reasons to continue smoking, which corresponds to our results. Additionally, addiction and habit were also mentioned as important reasons to continue smoking. However, our study did not confirm their result, that depressive symptoms were correlated with continued smoking, possibly due to the low prevalence of depressive symptoms in our sample. Besides above mentioned findings, concepts of addiction and habit and a possible interaction with other risk behaviors (e.g. alcohol consumption) should be taken into consideration in further research. Because of the increased health risk of continued smoking, the high rate of motivated current smokers, and limited research in this field, further exploration of predictors and the development of programs to (remain) quit smoking for cancer survivors are needed.

\section{Alcohol consumption}

Among alcohol drinkers, more than one third drank more than recommended, and $18.7 \%$ preformed binge drinking (six or more servings a day, 1-3 times per month or even more frequently), which is considerably more than reported in other studies $[62,65,66]$. Possibly, people might not be aware of their excessive alcohol consumption and its long-term risk [9, 67, 68]. Earlier studies in older adults reported that alcohol consumption was related to positive sensations among older adults $[69,70]$. Our finding, that low self-efficacy was associated with higher alcohol consumption might possibly be explained by the difficulty of breaking a particular drinking habit, assuming that a substantial number of participants consumed more than recommended, and thus drank regularly, and as discussed above, alcohol consumption might be accompanied by positive short term consequences. Given the long-term health risks, an increase of awareness and knowledge about personal (excessive) alcohol consumption and its consequences should be pursued in cancer survivors. It should be considered that our sample included never-drinkers, social drinkers and excessive drinkers, who possibly could be regarded as distinct groups.

\section{Vegetable and fruit consumption}

Vegetable and fruit consumption were low in our sample, however, consistent or higher than in American cancer survivors $[1,16,71]$. Compared to European cancer survivors, especially vegetable consumption was considerably lower $[65,72,73]$. These low prevalence rates clearly demonstrate that the vegetable and fruit consumption can be greatly improved.

In nutrition recommendations and studies, vegetable and fruit consumption often are treated and presented as one single behavior, although there are differences in the prevalence and consumption of fruit and vegetables, e.g. in the Netherlands, vegetables are mostly a part of the main meals and fruit is often eaten as a snack between meals or as a desert. Our study showed only a small correlation between vegetable and fruit consumption and the factors associated with both behaviors were different, which advocates for treating vegetable and fruit consumption as two different types of behavior. A longer period after completing primary cancer treatment was correlated with a higher amount of vegetable consumption, but not with fruit consumption. The preparation of vegetables could take some effort, and possibly, cancer survivors might spend more effort in the preparation of meals including vegetables, the more time passed after the cancer treatment with possible side effects. Furthermore, the sense of taste could be affected during the cancer treatment and improve again afterwards. Possibly, this also could be a reason for a temporary change in diet. However, evidence is limited yet about correlates and predictors of vegetable and fruit consumption in cancer survivors.

In the present study, the strongest correlates in vegetable and fruit consumption were positive intentions, while being women and having a higher education were found to be correlated to meeting vegetable and fruit recommendation in other research [21]. Furthermore, we identified that more excessive alcohol drinkers and smokers were less likely to adhere to the fruit recommendation. The latter might be explained by assuming that smokers possibly smoke at times when nonsmokers eat fruit (e.g. 
during break times at work). These results confirm prior findings that risk behaviors among adults tend to cluster [74]. Moreover, it is shown that combinations or clustering of risk behaviors might be involved with additional health risks [75].

To disentangle separate determinants of vegetable and fruit consumption, more specific research is needed. In clinical practice, attention should be given to vegetable and fruit consumption to increase the intake in cancer survivors, preferably tailored to personal attitudes, selfefficiency expectations, and intentions.

\section{Adherence to recommendations}

In our study, the adherence to recommendations (Fig. 1) was overall more positive in comparison with other studies [1, 3, 22]. Higher scores on attitude, self-efficacy, and intention of some of the lifestyle behaviors were the strongest correlates with adherence to an increasing number of recommendations (Table 6). The strong association between self-efficacy toward nonsmoking and adherence to recommendations could be explained by the presence of never-smokers (43.2\%) in our sample.

Not much is known about contributing factors in explaining adherence to an increasing number of lifestyle recommendations in cancer survivors, yet. We found that positive mental adjustment contributed $(p=.045)$, what could be in line with findings from other research, reporting that emotional benefit-finding related to cancer was positively associated with engagement in several health behaviors [76]. Although the two concepts are not the same, we could assume that cancer survivors who are able to cope more positively with their situation might be more likely to be involved in healthier lifestyle behaviors. However, a direction en causality of this association cannot be determined in this study. We emphasize again, that especially for cancer survivors it may be important to live as healthy as possible. Therefore, more insight is needed in the determinants of engagement in as much as possible healthy lifestyle behaviors, and, furthermore, cancer aftercare programs should aim to target multiple lifestyle behaviors.

\section{Different patterns of correlates}

For each separate lifestyle behavior we found different prevalence and different patterns of correlates. In accordance with the assumptions of social cognitive theories, we identified proximal variables and intention as strongest correlates in all examined behaviors, although with variations in contribution. Our results confirm theoretical assumptions [27], that the relative contribution of attitudes, selfefficacy and social influences can differ from one person to another and from one behavior to another. Regarding the distal factors, we found notably less, but also different patterns of correlations between the lifestyle behaviors.
Overall, subscales of the EORTC QLQ-C30 provided the most influential distal factors, although the contribution of all distal factors (socio-demographic, cancer-related, psychological) was considerably lower than the contribution of the proximal factors and intention. It would be interesting to investigate a possible predicting role of the distal factors and possible mediation effects of the proximal factors in longitudinal research.

\section{Limitations}

This study was subject to some limitations. Due to the cross-sectional design, no causal relationships and directions of associations could be determined. Furthermore, the collected data were based on self-report questionnaires. In particular, self-reported outcomes of lifestyle behaviors should be interpreted carefully. In addition, the results of his study might not be generalizable to all cancer survivors, because more than half of the sample has been women with breast cancer. Even though, cancer type and gender had limited correlates in explaining the lifestyle behaviors.

In measuring physical activity using IPAQ short form, possibly over reporting might have been occurred. This is known as a typical problem in several previous studies using the same questionnaire [77]. In this study, the cutoff point to achieve the physical activity recommendations was $600 \mathrm{MET}$-min/week, which is in accordance with the scorings guideline of the IPAQ questionnaire. However, in guidelines, different cut-off points or ranges were indicated [78-80]. Our cut-off point choice might have affected the outcome of the adherence to physical activity recommendations.

With regard to alcohol consumption, it could be that the results on alcohol are more a reflection of social drinkers and excessive drinkers, because some questions were focused on alcohol consumption, and non-drinkers might have found them to be not applicable to themselves. Although, similar questions were also applied to non-drinkers in prior research [81].

There was a probability that significant correlates could have occurred by chance due to multiple testing. However, by applying sequential multiple linear/logistic regression analyses, the chance on Type 1 error was rather small [58]. Moreover, given the adequate power, the $p$-values were highly significant which indicated that our estimates were relatively accurate.

\section{Conclusions}

Overall, the participants of our study were more engaged in healthy lifestyle behaviors compared to other research, however, especially vegetable and fruit consumption were poor and should be considerably improved. The various lifestyle behaviors and the adherence to recommendations were influenced by different patterns of correlates, from 
which self-efficacy, attitudes, and intention were the strongest, although their contribution varied among the different lifestyle behaviors. Our findings emphasized that all examined lifestyle behaviors need to be encouraged in cancer survivors, with taken into consideration that each lifestyle behavior is influenced by a specific set of mainly motivational correlates.

\section{Abbreviations}

ASE: Attitude-Social influence-Efficacy; BMI: body mass index; EORTC QLQ: European Organisation for Research and Treatment of Cancer; QoL: quality of life; HADS: Hospital Anxiety and Depression Scale; MAC: Mental Adjustment to Cancer scale; IPQ-R: Illness Perception Questionnaire Revised; SPSIR-R: S Short Social Problem Solving InventoryRevised; IPAQ: International Physical Activity Questionnaire; MET: Metabolic Equivalent of Task

\section{Competing interests}

The authors declare that they have no competing interests.

\section{Authors' contributions}

CAWB, IM, RAW, AAJMB and LL contributed to conceptualization and design of this study. RAW and AAJMB were involved in the acquisition of data. IMK and CAWB were involved in analysis and interpretation of data. IMK drafted the manuscript and all authors were involved in revising it critically, and read and approved the final manuscript.

\section{Acknowledgements}

This research project is funded by the Dutch Cancer Society (grant number NOU2011-5151). Our sincere thanks go out to all the Dutch hospitals who helped with the recruitment of the participants: Bernhoven hospital (Veghel), Catherina hospital (Eindhoven), Elkerliek hospital (Helmond), Jeroen Bosch hospital ('s-Hertogenbosch), Laurentius hospital (Roermond), Lievensberg hospital (Bergen op Zoom), Sint Anna hospital (Geldrop), and Zuyderland Medical Centre (Sittard). Moreover, we would like to thank Linda Küsters for her contribution to setting up the study.

\section{Author details}

${ }^{1}$ Faculty of Psychology and Educational Sciences, Open University of the Netherlands, P. O. Box 29606401DL, Heerlen, The Netherlands. ${ }^{2}$ CAPHRI School for Public Health and Primary Care, Maastricht University, P.O. Box 6166200 MD Maastricht, Maastricht, The Netherlands.

Received: 17 December 2014 Accepted: 15 December 2015 Published online: 05 January 2016

\section{References}

1. Blanchard CM, Courneya KS, Stein K. Cancer survivors' adherence to lifestyle behavior recommendations and associations with health-related quality of life: Results from the American Cancer Society's SCS-II. J Clin Oncol. 2008; doi:10.1200/JCO.2007.14.6217

2. Davies NJ, Batehup L, Thomas R. The role of diet and physical activity in breast, colorectal, and prostate cancer survivorship: a review of the literature. Br J Cancer. 2011; doi 10.1038/bjc.2011.423.

3. Schlesinger S, Walter J, Hampe J, von Schonfels W, Hinz S, Kuchler T, Jacobs G, Schafmayer C, Nothlings U. Lifestyle factors and health-related quality of life in colorectal cancer survivors. Cancer Causes Control. 2014; doi: 10.1007/ s10552-013-0313-y.

4. Ford ES, Bergmann MM, Kroger J, Schienkiewitz A, Weikert C, Boeing $H$. Healthy living is the best revenge: findings from the European Prospective Investigation Into Cancer and Nutrition-Potsdam study. Arch Intern Med. 2009; doi: 10.1001/archinternmed.2009.237.

5. Blanchard CM, Stein K, Baker F, Dent MF, Denniston MM, Courneya KS, Nehl E. Association between current lifestyle behaviors and health-related quality of life in breast, colorectal, and prostate cancer survivors. Psychol Health 2004; doi: 10.1080/08870440310001606507.

6. Baena Ruiz R, Salinas Hernandez P: Diet and cancer: Risk factors and epidemiological evidence. Maturitas. 2014; doi:10.1016/j.maturitas.2013.11.010
7. Kushi LH, Kwan ML, Lee MM, Ambrosone CB. Lifestyle factors and survival in women with breast cancer. J Nutr. 2007:137:236S-42S.

8. Colditz GA, Wolin KY, Gehlert S. Applying what we know to accelerate cancer prevention. Sci Transl Med. 2012; doi: 10.1126/scitransImed.3003218.

9. McLaughlin VH, Trentham-Dietz A, Hampton JM, Newcomb PA, Sprague BL. Lifestyle factors and the risk of a second breast cancer after ductal carcinoma in situ. Cancer Epidemiol Biomarkers Prev. 2014; doi:10.1158/ 1055-9965.EPI-13-0899.

10. Inoue-Choi M, Robien K, Lazovich D. Adherence to the WCRF/AICR guidelines for cancer prevention is associated with lower mortality among older female cancer survivors. Cancer Epidemiol Biomarkers Prev. 2013; doi: 10.1158/1055-9965.EPI-13-0054.

11. Schmid D, Leitzmann MF. Association between physical activity and mortality among breast cancer and colorectal cancer survivors: a systematic review and meta-analysis. Ann Oncol. 2014;doi: 10.1093/annonc/mdu012.

12. Klosky JL, Tyc VL, Garces-Webb DM, Buscemi J, Klesges RC, Hudson MM. Emerging issues in smoking among adolescent and adult cancer survivors. A comprehensive review. Cancer. 2007; doi: 10.1002/cncr.23061

13. Wilson D, Parsons J, Wakefield $M$. The health-related quality-of-life of never smokers, ex-smokers, and light, moderate, and heavy smokers. Prev Med. 1999; doi: 10.1006/pmed.1999.0523.

14. Rock CL, Doyle C, Demark-Wahnefried W, Meyerhardt J, Courneya KS, Schwartz AL, Bandera EV, Hamilton KK, Grant B, McCullough M, et al. Nutrition and physical activity guidelines for cancer survivors. CA Cancer J Clin. 2012; doi: 10.3322/caac.21142

15. Sehl M, Lu X, Silliman R, Ganz PA. Decline in physical functioning in first 2 years after breast cancer diagnosis predicts 10-year survival in older women. J Cancer Surviv. 2013; doi: 10.1007/s11764-012-0239-5.

16. LeMasters TJ, Madhavan SS, Sambamoorthi U, Kurian S. Health behaviors among breast, prostate, and colorectal cancer survivors: a US population-based case-control study, with comparisons by cancer type and gender. J Cancer Surviv. 2014; doi: 10.1007/s11764-014-0347-5.

17. Bellizzi KM, Rowland JH, Jeffery DD, McNeel T. Health behaviors of cancer survivors: examining opportunities for cancer control intervention. J Clin Oncol. 2005; doi: 10.1200/jco.2005.02.2343.

18. Williams K, Steptoe A, Wardle J. Is a cancer diagnosis a trigger for health behaviour change? Findings from a prospective, population-based study. $\mathrm{Br}$ J Cancer. 2013; doi: 10.1038/bjc.2013.254.

19. Coups EJ, Ostroff JS. A population-based estimate of the prevalence of behavioral risk factors among adult cancer survivors and noncancer controls. PrevMed. 2005; doi: 10.1016/j.ypmed.2004.09.011.

20. Eakin EG, Youlden DR, Baade PD, Lawler SP, Reeves MM, Heyworth JS, Fritschi L. Health behaviors of cancer survivors: data from an Australian population-based survey. Cancer Causes Control. 2007; doi: DOl10.1007/ s10552-007-9033-5 18:881-894

21. Mayer DK, Terrin NC, Menon U, Kreps GL, McCance K, Parsons SK, Mooney $\mathrm{KH}$. Health behaviors in cancer survivors. Oncol Nurs Forum. 2007; doi: 10. 1188/07.ONF.643-651.

22. O'Neill SC, DeFrank JT, Vegella P, Richman AR, Henry LR, Carey LA, Brewer NT. Engaging in health behaviors to lower risk for breast cancer recurrence. PloS One. 2013; doi: 10.1371/journal.pone.0053607.

23. Pronk NP, Anderson LH, Crain AL, Martinson BC, O'Connor PJ, Sherwood NE, Whitebird RR. Meeting recommendations for multiple healthy lifestyle factors. Prevalence, clustering, and predictors among adolescent, adult, and senior health plan members. Am J Prev Med. 2004; doi:10.1016/j.amepre.2004.04.022.

24. De Vries H, Mudde AN. Predicting stage transitions for smoking cessation applying the attitude-social influence-efficacy model. Psychol Health. 1998;13: 369-85.

25. De Vries H, Mudde AN, Dijkstra A, Willemsen MC. Differential beliefs, perceived social influences, and self-efficacy expectations among smokers in various motivational phases. Prev Med. 1998; doi: 10.1006/pmed.1998.0344.

26. De Vries H, Mudde AN, Leijs I, Charlton A, Vartiainen E, Buijs G, et al. The European smoking prevention framework approach (EFSA): An example of integral prevention. Health Educ Res. 2003;18:611-26.

27. Fishbein M, Ajzen I. Predicting And Changing Behavior. The Reasoned Action Approach. New York: Tyler and Francis Group, LLC; 2010.

28. Charlier C, Van Hoof E, Pauwels E, Lechner L, Spittaels H, De Bourdeaudhuij I. The contribution of general and cancer-related variables in explaining physical activity in a breast cancer population 3 weeks to 6 months posttreatment. Psychooncology. 2013; doi: 10.1002/pon.2079. 
29. Forbes CC, Blanchard CM, Mummery WK, Courneya KS. A comparison of physical activity correlates across breast, prostate and colorectal cancer survivors in Nova Scotia, Canada. Support Care Cancer. 2014;doi: 10.1007/ s00520-013-2045-7.

30. Husebo AM, Dyrstad SM, Soreide JA, Bru E. Predicting exercise adherence in cancer patients and survivors: a systematic review and meta-analysis of motivational and behavioural factors. J Clin Nurs. 2013; doi: 10.1111/j.1365-2702.2012.04322.x.

31. Kampshoff CS, Jansen F, van Mechelen W, May AM, Brug J, Chinapaw MJ, Buffart LM. Determinants of exercise adherence and maintenance among cancer survivors: a systematic review. Int J Behav Nutr Phys Act. 2014; doi: 10.1186/1479-5868-11-80

32. Madlensky L, Natarajan L, Flatt SW, Faerber S, Newman VA, Pierce JP. Timing of dietary change in response to a telephone counseling intervention: evidence from the WHEL study. Health Psychol. 2008; doi: 10.1037/0278 6133.27.5.539.

33. Westmaas JL, Alcaraz Kl, Berg CJ, Stein KD. Prevalence and Correlates of Smoking and Cessation-Related Behavior among Survivors of Ten Cancers: Findings from a Nationwide Survey Nine Years after Diagnosis. Cancer Epidemiol Biomarkers Prev. 2014; doi: 10.1158/1055-9965.EPI-14-0046.

34. American Psychological Association. Ethical Principles of Psychologists and Code of Conduct. http://www.apa.org/ethics/code/index.aspx (2010). Accessed $10 \mathrm{dec}$ 2015.

35. Craig $C L$, Marshall AL, Sjostrom M, Bauman AE, Booth ML, Ainsworth BE, Pratt M, Ekelund U, Yngve A, Sallis JF, Oja P. International physical activity questionnaire: 12-country reliability and validity. Med Sci Sports Exerc. 2003; doi: 10.1249/01.MSS.0000078924.61453.FB.

36. The International Physical Activity Questionnaire. https://sites.google.com/ site/theipaq/. Accessed 8 march 2014.

37. Vandelanotte C, De Bourdeaudhuij I, Philippaerts R, Sjöström M, Sallis J. Reliability and validity of a computerized and Dutch version of the International Physical Activity Questionnaire (IPAQ). J Phys Act Health. 2005; 2:63.

38. Mudde AN, Willemsen MC, Kremers S, De Vries H. Meetinstrumenten voor onderzoek naar roken en stoppen met roken. Den Haag: Stivoro; 2006.

39. Loon AJM van, Veldhuizen H. Voortgangsrapportage 2003. Lokale en Nationale Monitor Volksgezondheid. http://www.rivm.nl/Documenten_en_ publicaties/Wetenschappelijk/Rapporten/2004/augustus/

Voortgangsrapportage_2003_Lokale_en_Nationale_Monitor_ Volksgezondheid?sp=cml2bXE9ZmFsc2U7c2VhcmNoYmFzZTOz NDQ4MDtyaXZtcT1mYWxzZTs=\&pagenr=3449. Accessed 8 march 2014.

40. Brink $C L$ van den, Ocke MC, Houben AW, Nierop P van, Droomers M. Validering van standaardvraagstelling voeding voor Lokale en Nationale Monitor Volksgezondheid. 2005. http://www.rivm.nl/Documenten_en_ publicaties/Wetenschappelijk/Rapporten/2005/augustus/Nalidering_van_ standaardvraagstelling_voeding_voor_Lokale_en_Nationale_Monitor_ Volksgezondheid. Accessed 8 march 2014

41. Aaronson NK, Ahmedzai S, Bergman B, Bullinger M, Cull A, Duez NJ, et al. The European Organization for Research and Treatment of Cancer QLQ-C30: A quality-of-life instrument for use in international clinical trials in oncology. J Natl Cancer Inst. 1992;85:365-76.

42. Fayers P, Bottomley A. Quality of life research within the EORTC - the EORTC QLQ-C30. Eur J Cancer. 2002; doi: 10.1016/s0959-8049(01)00448-8.

43. Fayers PM, Aaronson NK, Bjordal K, Groenvold M, Curran D, Bottomly A, et al. The EORTC QLQ-C30 Scoring Manual. 3rd ed. Brussels: European Organisation for Research and Treatment of Cancer; 2001. http://www. eortc.be/qol/files/SCManualQLQ-C30.pdf.

44. Bjelland I, Dahl AA, Haug T, Neckelmann D. The validity of the Hospital Anxiety and Depression Scale. An updated literature review. J Psychosom Res. 2002:52:69-77.

45. Zigmond AS, Snaith RP. The hospital anxiety and depression scale. Acta Psychiatr Scand. 1983;67:361-70.

46. Mitchell AJ, Meader N, Symonds P. Diagnostic validity of the Hospital Anxiety and Depression Scale (HADS) in cancer and palliative settings: a meta-analysis. J Affect Disord. 2010; doi 10.1016/j.jad.2010.01.067.

47. Braeken AP, Kempen Gl, Watson M, Houben RM, Gils FC, Lechner L. Psychometric properties of the Dutch version of the Mental Adjustment to Cancer scale in Dutch cancer patients. Psychooncology. 2010; doi: 10.1002/pon.1628.

48. Watson M, Greer S, Young J, Inayat Q, Burgess C, Robertson B. Development of a questionnaire measure of adjustment to cancer: the MAC scale. Psychol Med. 1988; doi: 10.1007/s10549-005-9018-6.
49. Watson M, Homewood J. Mental Adjustment to Cancer Scale: psychometric properties in a large cancer cohort. Psychooncology. 2008; doi: 10.1002/pon.1345.

50. Broadbent E, Petrie KJ, Main J, Weinman J. The brief illness perception questionnaire. J Psychosom Res. 2006; doi: 10.1016/j.jpsychores.2005.10.020.

51. Weinman J, Petrie KJ, Moss-Morris R, Horne R. The illness perception questionnaire: A new method for assessing the cognitive representation of illness. Psychol Health. 1996; doi: 10.1080/08870449608400270.

52. D'Zurilla TJ, Nezu AM, Maydeu-Olivares A. Social Problem-Solving Inventory - Revised. 2007. http://www.mhs.com/product.aspx?gr=cli\&prod=spsir\&id=overview\#top. Accessed 8 march 2014.

53. Smeets T, Kremers SP, Brug J, de Vries H. Effects of tailored feedback on multiple health behaviors. Ann Behav Med. 2007; doi: 10.1093/her/cyl10.

54. Smit ES, de Vries $H$, Hoving C. Determinants of practice nurses' intention to implement a new smoking cessation intervention: the importance of attitude and innovation characteristics. J Adv Nurs. 2013; doi: 10.1111/jan.12153.

55. Bakker EC, Nijkamp MD, Sloot C, Berndt NC, Bolman CA. Intention to abstain from smoking among cardiac rehabilitation patients: the role of attitude, self-efficacy, and craving. J Cardiovasc Nurs. 2015; doi: 10.1097/JCN. 0000000000000156.

56. Berndt N, Bolman C, Froelicher ES, Mudde A, Candel M, de Vries $\mathrm{H}$, Lechner L. Effectiveness of a telephone delivered and a face-to-face delivered counseling intervention for smoking cessation in patients with coronary heart disease: a 6-month follow-up. J Behav Med. 2014; doi: 10.1007/s10865-013-9522-9.

57. Ronda G, Van Assema P, Brug J. Stages of change, psychological factors and awareness of physical activity levels in The Netherlands. Health Promot Int. 2001;16:305-14.

58. Tabachnick BG, Fidell LS. Using Multivariate Statistics. 4th ed. Boston, London, Toronto, Sydney, Tokyo, Singapore: Allyn and Bacon; 2001.

59. Stevinson C, Lydon A, Amir Z. Adherence to physical activity guidelines among cancer support group participants. Eur J Cancer Care. 2014; doi: 10. 1111/ecc.12145

60. Bertheussen GF, Oldervoll L, Kaasa S, Sandmael JA, Helbostad JL. Measurement of physical activity in cancer survivors-a comparison of the HUNT 1 Physical Activity Questionnaire (HUNT 1 PA-Q) with the International Physical Activity Questionnaire (IPAQ) and aerobic capacity. Support Care Cancer. 2013; doi: 10.1007/s00520-012-1530-8.

61. Cuevas BT, Hughes DC, Parma DL, Trevino-Whitaker RA, Ghosh S, Li R, Ramirez AG. Motivation, exercise, and stress in breast cancer survivors. Support Care Cancer. 2014; doi: 10.1007/s00520-013-2038-6.

62. Del Valle MO, Martin-Payo R, Lana A, Garcia JB, Folgueras MV, Lopez ML. Behavioural cancer risk factors in women diagnosed with primary breast cancer. J Adv Nurs. 2014; doi: 10.1111/jan.12433.

63. Weaver KE, Foraker RE, Alfano CM, Rowland JH, Arora NK, Bellizzi KM, Hamilton AS, Oakley-Girvan I, Keel G, Aziz NM. Cardiovascular risk factors among long-term survivors of breast, prostate, colorectal, and gynecologic cancers: a gap in survivorship care? J Cancer Surviv. 2013; doi: 10.1007/ s11764-013-0267-9.

64. Berg CJ, Thomas AN, Mertens AC, Schauer GL, Pinsker EA, Ahluwalia JS, Khuri FR. Correlates of continued smoking versus cessation among survivors of smoking-related cancers. Psychooncology. 2012; doi: 10.1002/pon.3077.

65. Grimmett C, Bridgewater J, Steptoe A, Wardle J: Lifestyle and quality of life in colorectal cancer survivors. Qual Life Res. 2011; doi: 10.1007/s11136-011-9855-1.

66. Bidstrup PE, Dalton SO, Christensen J, Tjonneland A, Larsen SB, Karlsen R, Brewster A, Bondy M, Johansen C. Changes in body mass index and alcohol and tobacco consumption among breast cancer survivors and cancer-free women: a prospective study in the Danish Diet, Cancer and Health Cohort. Acta Oncol. 2013; doi: 10.3109/0284186X.2012.746466.

67. Dumalaon-Canaria JA, Hutchinson AD, Prichard I, Wilson C. What causes breast cancer? A systematic review of causal attributions among breast cancer survivors and how these compare to expert-endorsed risk factors. Cancer Causes Control. 2014; doi: 10.1007/s10552-014-0377-3.

68. Kwan ML, Kushi LH, Weltzien E, Tam EK, Castillo A, Sweeney C, Caan BJ. Alcohol consumption and breast cancer recurrence and survival among women with early-stage breast cancer: the life after cancer epidemiology study. J Clin Oncol. 2010; doi: 10.1200/JCO.2010.29.2730.

69. Chan AM, von Muhlen D, Kritz-Silverstein D, Barrett-Connor E. Regular alcohol consumption is associated with increasing quality of life and mood in older men and women: the Rancho Bernardo Study. Maturitas. 2009; doi: 10.1016/j.maturitas.2009.01.005. 
70. Lang I, Wallace RB, Huppert FA, Melzer D. Moderate alcohol consumption in older adults is associated with better cognition and well-being than abstinence. Age Ageing. 2007; doi: 10.1093/ageing/afm001.

71. Milliron BJ, Vitolins MZ, Tooze JA. Usual Dietary Intake among Female Breast Cancer Survivors Is Not Significantly Different from Women with No Cancer History: Results of the National Health and Nutrition Examination Survey, 2003-2006. J Acad Nutr Diet. 2013; doi: 10.1016/j.jand.2013.08.015.

72. Ocke MC, Buurma-Rethans EJM, Boer de EJ, Wilson-van den Hooven C, Etemad-Ghameshlou Z, Drijvers JJMM, Rossum van CTM. Diet of community-dwelling ouder adults. Dutch National Food Consumption Survey Older adults 2010-2012. http://www.rivm.nl/Documenten_en_ publicaties/Wetenschappelijk/Rapporten/2013/oktober/Diet_of_community_ dwelling_older_adults_Dutch_National_Food_Consumption_Survey_Older_ adults_2010_2012. Accessed 18 jan 2014.

73. Dijkstra SC, Neter JE, Brouwer IA, Huisman M, Visser M. Adherence to dietary guidelines for fruit, vegetables and fish among older Dutch adults; the role of education, income and job prestige. J Nutr Health Aging. 2014; doi: 10. 1007/s12603-013-0402-3.

74. Spring B, King AC, Pagoto SL, Van Horn L, Fisher JD. Fostering multiple healthy lifestyle behaviors for primary prevention of cancer. Am Psychol. 2015; doi: 10.1037/a0038806.

75. Bradbury KE, Appleby PN, Key TJ. Fruit, vegetable, and fiber intake in relation to cancer risk: findings from the European Prospective Investigation into Cancer and Nutrition (EPIC). Am J Clin Nutr. 2014; doi: 10.3945/ajcn.113. 071357.

76. Low CA, Beckjord E, Bovbjerg DH, Dew MA, Posluszny DM, Schmidt JE, Lowery AE, Nutt SA, Arvey SR, Rechis R. Correlates of positive health behaviors in cancer survivors: Results from the 2010 LIVESTRONG Survey. J Psychosoc Oncol. 2014; doi: 10.1080/07347332.2014.955243.

77. Lee PH, Macfarlane DJ, Lam TH, Stewart SM. Validity of the International Physical Activity Questionnaire Short Form (IPAQ-SF): a systematic review. Int J Behav Nutr Phys Act. 2011; doi: 10.1186/1479-5868-8-115

78. Haskell WL, Lee IM, Pate RR, Powell KE, Blair SN, Franklin BA, Macera CA, Heath GW, Thompson PD, Bauman A. Physical activity and public health: updated recommendation for adults from the American College of Sports Medicine and the American Heart Association. Circulation. 2007; doi: 10. 1161/CIRCULATIONAHA. 107.185649.

79. Nelson ME, Rejeski WJ, Blair SN, Duncan PW, Judge JO, King AC, Macera CA, Castaneda-Sceppa C. Physical activity and public health in older adults: recommendation from the American College of Sports Medicine and the American Heart Association. Circulation. 2007; doi:10.1161/ CIRCULATIONAHA.107.185650.

80. Garber CE, Blissmer B, Deschenes MR, Franklin BA, Lamonte MJ, Lee IM, Nieman DC, Swain DP. American College of Sports Medicine position stand. Quantity and quality of exercise for developing and maintaining cardiorespiratory, musculoskeletal, and neuromotor fitness in apparently healthy adults: guidance for prescribing exercise. Med Sci Sports Exerc. 2011: doi: 10.1249/MSS.0b013e318213fefb.

81. Schulz DN, Kremers SP, Vandelanotte C, van Adrichem MJ, Schneider F, Candel MJ, de Vries H. Effects of a web-based tailored multiple-lifestyle intervention for adults: a two-year randomized controlled trial comparing sequential and simultaneous delivery modes. J Med Internet Res 2014; doi: 10.2196/jmir.3094

\section{Submit your next manuscript to BioMed Centra and we will help you at every step:}

- We accept pre-submission inquiries

- Our selector tool helps you to find the most relevant journal

- We provide round the clock customer support

- Convenient online submission

- Thorough peer review

- Inclusion in PubMed and all major indexing services

- Maximum visibility for your research

Submit your manuscript at www.biomedcentral.com/submit 